\title{
Common Solutions of Generalized Mixed Equilibrium Problems, Variational Inclusions, and Common Fixed Points for Nonexpansive Semigroups and Strictly Pseudocontractive Mappings
}

\author{
Poom Kumam, ${ }^{1}$ Usa Hamphries, ${ }^{1}$ and Phayap Katchang ${ }^{2}$ \\ ${ }^{1}$ Department of Mathematics, Faculty of Science, King Mongkut's University of Technology Thonburi \\ (KMUTT), Bangmod, Bangkok 10140, Thailand \\ ${ }^{2}$ Department of Mathematics and Statistics, Faculty of Science and Agricultural Technology, Rajamangala \\ University of Technology Lanna Tak, Tak 63000, Thailand
}

Correspondence should be addressed to Phayap Katchang, p.katchang@hotmail.com

Received 24 April 2011; Accepted 12 July 2011

Academic Editor: Yansheng Liu

Copyright (c) 2011 Poom Kumam et al. This is an open access article distributed under the Creative Commons Attribution License, which permits unrestricted use, distribution, and reproduction in any medium, provided the original work is properly cited.

We introduce a new iterative scheme by shrinking projection method for finding a common element of the set of solutions of generalized mixed equilibrium problems, the set of common solutions of variational inclusion problems with set-valued maximal monotone mappings and inverse-strongly monotone mappings, the set of solutions of fixed points for nonexpansive semigroups, and the set of common fixed points for an infinite family of strictly pseudocontractive mappings in a real Hilbert space. We prove that the sequence converges strongly to a common element of the above four sets under some mind conditions. Furthermore, by using the above result, an iterative algorithm for solution of an optimization problem was obtained. Our results improve and extend the corresponding results of Martinez-Yanes and Xu (2006), Shehu (2011), Zhang et al. (2008), and many authors.

\section{Introduction}

Throughout this paper, we assume that $H$ is a real Hilbert space with inner product and norm are denoted by $\langle\cdot, \cdot\rangle$ and $\|\cdot\|$, respectively. Let $2^{H}$ denote the family of all subsets of $H$, and let $C$ be a closed-convex subset of $H$. Recall that a mapping $T: C \rightarrow C$ is said to be a $k$-strict pseudocontraction [1] if there exists $0 \leq k<1$ such that

$$
\|T x-T y\|^{2} \leq\|x-y\|^{2}+k\|(I-T) x-(I-T) y\|^{2}, \quad \forall x, y \in C,
$$


where $I$ denotes the identity operator on $C$. When $k=0, T: C \rightarrow C$ is said to be nonexpansive [2] if

$$
\|T x-T y\| \leq\|x-y\|, \quad \forall x, y \in C
$$

And when $k=1, T: C \rightarrow C$ is said to be pseudocontraction if

$$
\|T x-T y\|^{2} \leq\|x-y\|^{2}+\|(I-T) x-(I-T) y\|^{2}, \quad \forall x, y \in C .
$$

Clearly, the class of $k$-strict pseudocontraction falls into the one between classes of nonexpansive mappings and pseudocontraction mapping. We denote the set of fixed points of $T$ by $F(T)$.

A family $\mathcal{S}=\{S(s): 0 \leq s<\infty\}$ of mappings of $C$ into itself is called a nonexpansive semigroup on $C$ if it satisfies the following conditions:

(i) $S(0) x=x$ for all $x \in C$,

(ii) $S(s+t)=S(s) S(t)$ for all $s, t \geq 0$,

(iii) $\|S(s) x-S(s) y\| \leq\|x-y\|$ for all $x, y \in C$ and $s \geq 0$,

(iv) for all $x \in C, s \mapsto S(s) x$ is continuous.

We denote by $F(\mathcal{S})$ the set of all common fixed points of $\mathcal{S}=\{S(s): s \geq 0\}$, that is, $F(S)=$ $\bigcap_{s \geq 0} F(S(s))$. It is known that $F(S)$ is closed and convex.

Let $A: H \rightarrow H$ be a single-valued nonlinear mapping, and let $M: H \rightarrow 2^{H}$ be a set-valued mapping. We consider the following variational inclusion problem, which is to find a point $u \in H$ such that

$$
\theta \in A(u)+M(u)
$$

where $\theta$ is the zero vector in $H$. The set of solutions of problem (1.4) is denoted by $I(A, M)$.

Let the set-valued mapping $M: H \rightarrow 2^{H}$ be a maximal monotone. We define the resolvent operator $J_{M, \lambda}$ associate with $M$ and $\lambda$ as follows:

$$
J_{M, \lambda}(u)=(I+\lambda M)^{-1}(u), \quad u \in H
$$

where $\lambda$ is a positive number. It is worth mentioning that the resolvent operator $J_{M, \lambda}$ is singlevalued, nonexpansive, and 1-inverse-strongly monotone $([3,4])$.

Let $F$ be a bifunction of $C \times C$ into $\mathbb{R}$, where $\mathbb{R}$ is the set of real numbers, let $A: C \rightarrow H$ be a mapping, and let $\varphi: C \rightarrow \mathbb{R}$ be a real-valued function. The generalized mixed equilibrium problem is for finding $x \in C$ such that

$$
F(x, y)+\langle A x, y-x\rangle+\varphi(y)-\varphi(x) \geq 0, \quad \forall y \in C
$$


The set of solutions of (1.6) is denoted by $\operatorname{GMEP}(F, \varphi, A)$, that is,

$$
\operatorname{GMEP}(F, \varphi, A)=\{x \in C: F(x, y)+\langle A x, y-x\rangle+\varphi(y)-\varphi(x) \geq 0, \forall y \in C\} .
$$

If $A \equiv 0$, then the problem (1.6) is reduced into the mixed equilibrium problem for finding $x \in C$ such that

$$
F(x, y)+\varphi(y)-\varphi(x) \geq 0, \quad \forall y \in C .
$$

The set of solutions of (1.8) is denoted by $\operatorname{MEP}(F, \varphi)$. The (generalized) mixed equilibrium problems include fixed-point problems, variational inequality problems, optimization problems, Nash equilibrium problems, noncooperative games, economics, and the equilibrium problem as special cases ([5-15]). In the last two decades, many papers have appeared in the literature on the existence of solutions of equilibrium problems; see, for example, [9] and references therein. Some solution methods have been proposed to solve the mixed equilibrium problems; see, for example, ([7-10, 12-20]) and references therein.

In 2006, Martinez-Yanes and Xu [21] introduced the following iterative:

$$
\begin{gathered}
x_{0} \in C, \\
y_{n}=\alpha_{n} x_{0}+\left(1-\alpha_{n}\right) T x_{n} \\
C_{n}=\left\{z \in C:\left\|y_{n}-z\right\|^{2} \leq\left\|x_{n}-z\right\|^{2}+\alpha_{n}\left(\left\|x_{0}\right\|^{2}+2\left\langle x_{n}-x_{0}, z\right\rangle\right)\right\}, \\
Q_{n}=\left\{z \in C:\left\langle x_{n}-z, x_{n}-x_{0}\right\rangle \leq 0\right\}, \\
x_{n+1}=P_{C_{n} \cap Q_{n}} x_{0},
\end{gathered}
$$

where $T$ is a nonexpansive mapping in a Hilbert space $H$, and $P_{C}$ is metric projection of $H$ onto a closed and convex subset $C$ of $H$. They proved that if the sequence $\left\{\alpha_{n}\right\}$ of parameters satisfies appropriate conditions, then the sequence $\left\{x_{n}\right\}$ converges strongly to $P_{F(T)} x_{0}$.

In 2008, Zhang et al. [4] introduced an iterative scheme for finding a common element of the set of solutions to the variational inclusion problem with a multivalued maximal monotone mapping and an inverse-strongly monotone mapping and the set of fixed points of nonexpansive mapping in Hilbert spaces. The following iterative scheme $x_{0}=x \in H$ and

$$
\begin{aligned}
& y_{n}=J_{M, \lambda}\left(x_{n}-\lambda A x_{n}\right), \\
& x_{n+1}=\alpha_{n} x+\left(1-\alpha_{n}\right) S y_{n},
\end{aligned}
$$

for all $n \geq 0$. They proved the strong convergence theorem under some mind conditions. 
Recently, Shehu [19] introduced a new iterative scheme by hybrid method for finding a common element of the set of common fixed points of infinite family of $k$-strictly pseudocontractive mappings, the set of common solutions to a system of generalized mixed equilibrium problems, and the set of solutions to a variational inequality problem in Hilbert spaces. Starting with an arbitrary $x_{0} \in C, C_{1, i}=C, C_{1}=\bigcap_{i=1}^{\infty} C_{1, i}$, and $x_{1}=P_{C_{1}} x_{0}$ define sequence $\left\{x_{n}\right\},\left\{w_{n}\right\},\left\{u_{n}\right\},\left\{z_{n}\right\}$, and $\left\{y_{n, i}\right\}$ as follows:

$$
\begin{gathered}
z_{n}=T_{r_{n}}^{\left(F_{1}, \varphi_{1}\right)}\left(x_{n}-r_{n} A x_{n}\right), \\
y_{n}=T_{\left.\lambda_{n}, \varphi_{2}\right)}^{\left(F_{2}\right)}\left(z_{n}-\lambda_{n} B z_{n}\right), \\
w_{n}=P_{C}\left(u_{n}-s_{n} D u_{n}\right), \\
y_{n, i}=\alpha_{n, i} w_{n}+\left(1-\alpha_{n, i}\right) T_{i} w_{n}, \quad n \geq 1, \\
C_{n+1, i}=\left\{z \in C_{n, i}:\left\|y_{n, i}-z\right\| \leq\left\|x_{n}-z\right\|\right\}, \quad n \geq 1, \\
C_{n+1}=\cap_{i=1}^{\infty} C_{n+1, i}, \\
x_{n+1}=P_{C_{n+1}} x_{0}, \quad n \geq 1,
\end{gathered}
$$

where $T_{i}$ is a $k_{i}$-strictly pseudocontractive mapping and for some $0 \leq k_{i}<1, A, B$ is $\alpha, \beta$-inverse-strongly monotone mapping of $C$ into $H$. He proved that if the sequence $\left\{\alpha_{n, i}\right\},\left\{r_{n}\right\},\left\{s_{n}\right\}$, and $\left\{\lambda_{n}\right\}$ of parameters satisfies appropriate conditions, then $\left\{x_{n}\right\}$ generated by (1.11) converges strongly to $P_{\Omega} x_{0}$.

In this paper, motivated by the above results, we present a new general iterative scheme for finding a common element of the set of solutions for a system of generalized mixed equilibrium problems, the set of common solutions of variational inclusion problems with set-valued maximal monotone mappings and inverse-strongly monotone mappings, the set of solutions of fixed points for nonexpansive semigroup mappings, and the set of common fixed points for an infinite family of strictly pseudocontractive mappings in a real Hilbert space. Then, we prove strong convergence theorem under some mind conditions. Furthermore, by using the above result, an iterative algorithm for solution of an optimization problem was obtained. The results presented in this paper extend and improve the results of Martinez-Yanes and Xu [21], Shehu [19], Zhang et al. [4], and many authors.

\section{Preliminaries}

Let $H$ be a real Hilbert space with norm $\|\cdot\|$ and inner product $\langle\cdot, \cdot\rangle$, and let $C$ be a closedconvex subset of $H$. When $\left\{x_{n}\right\}$ is a sequence in $H, x_{n} \rightarrow x$ means that $\left\{x_{n}\right\}$ converges weakly to $x$, and $x_{n} \rightarrow x$ means that $\left\{x_{n}\right\}$ converges strongly to $x$. In a real Hilbert space $H$, we have

$$
\begin{gathered}
\|x-y\|^{2}=\|x\|^{2}-\|y\|^{2}-2\langle x-y, y\rangle, \\
\|\lambda x+(1-\lambda) y\|^{2}=\lambda\|x\|^{2}+(1-\lambda)\|y\|^{2}-\lambda(1-\lambda)\|x-y\|^{2}, \quad \forall x, y \in H,
\end{gathered}
$$


and $\lambda \in \mathbb{R}$. For every point $x \in H$, there exists a unique nearest point in $C$, denoted by $P_{C} x$, such that

$$
\left\|x-P_{C} x\right\| \leq\|x-y\|, \quad \forall y \in C
$$

$P_{C}$ is called the metric projection of $H$ onto $C$. It is well known that $P_{C}$ is a nonexpansive mapping of $H$ onto $C$ and satisfies

$$
\left\langle x-y, P_{C} x-P_{C} y\right\rangle \geq\left\|P_{C} x-P_{C} y\right\|^{2}, \quad \forall x, y \in H
$$

Moreover, $P_{C} x$ is characterized by the following properties: $P_{C} x \in C$ and

$$
\begin{gathered}
\left\langle x-P_{C} x, y-P_{C} x\right\rangle \leq 0 \\
\|x-y\|^{2} \geq\left\|x-P_{C} x\right\|^{2}+\left\|y-P_{C} x\right\|^{2}, \quad \forall x \in H, y \in C .
\end{gathered}
$$

Recall that a mapping $A$ of $H$ into itself is called $\alpha$-inverse-strongly monotone if there exists a positive real number $\alpha$ such that

$$
\langle A x-A y, x-y\rangle \geq \alpha\|A x-A y\|^{2}, \quad \forall, y \in H
$$

It is obvious that any $\alpha$-inverse-strongly monotone mapping $A$ is $(1 / \alpha)$-Lipschitz monotone and continuous mapping.

In order to prove our main results, we need the following Lemmas.

Lemma 2.1 (see [22]). Let $V: C \rightarrow H$ be a $k$-strict pseudocontraction, then

(1) the fixed-point set $F(V)$ of $V$ is closed convex, so that the projection $P_{F(V)}$ is well defined;

(2) define a mapping $T: C \rightarrow H$ by

$$
T x=t x+(1-t) V x, \quad \forall x \in C .
$$

If $t \in[k, 1)$, then $T$ is a nonexpansive mapping such that $F(V)=F(T)$.

A family of mappings $\left\{V_{i}: C \rightarrow H\right\}_{i=1}^{\infty}$ is called a family of uniformly $k$-strict pseudocontractions if there exists a constant $k \in[0,1)$ such that

$$
\left\|V_{i} x-V_{i} y\right\|^{2} \leq\|x-y\|^{2}+k\left\|\left(I-V_{i}\right) x-\left(I-V_{i}\right) y\right\|^{2}, \quad \forall x, y \in C, \forall i \geq 1 .
$$


Let $\left\{V_{i}: C \rightarrow C\right\}_{i=1}^{\infty}$ be a countable family of uniformly $k$-strict pseudocontractions. Let $\left\{T_{i}: C \rightarrow C\right\}_{i=1}^{\infty}$ be the sequence of nonexpansive mappings defined by (2.8), that is,

$$
T_{i} x=t x+(1-t) V_{i} x, \quad \forall x \in C, \forall i \geq 1, t \in[k, 1) .
$$

Let $\left\{T_{i}\right\}$ be a sequence of nonexpansive mappings of $C$ into itself defined by (2.10), and let $\left\{\mu_{i}\right\}$ be a sequence of nonnegative numbers in $[0,1]$. For each $n \geq 1$, define a mapping $W_{n}$ of $C$ into itself as follows:

$$
\begin{aligned}
U_{n, n+1} & =I, \\
U_{n, n} & =\mu_{n} T_{n} U_{n, n+1}+\left(1-\mu_{n}\right) I, \\
U_{n, n-1} & =\mu_{n-1} T_{n-1} U_{n, n}+\left(1-\mu_{n-1}\right) I, \\
& \vdots \\
U_{n, k} & =\mu_{k} T_{k} U_{n, k+1}+\left(1-\mu_{k}\right) I, \\
U_{n, k-1} & =\mu_{k-1} T_{k-1} U_{n, k}+\left(1-\mu_{k-1}\right) I, \\
& \vdots \\
U_{n, 2} & =\mu_{2} T_{2} U_{n, 3}+\left(1-\mu_{2}\right) I, \\
W_{n}=U_{n, 1} & =\mu_{1} T_{1} U_{n, 2}+\left(1-\mu_{1}\right) I .
\end{aligned}
$$

Such a mapping $W_{n}$ is nonexpansive from $C$ to $C$ and it is called the $W$-mapping generated by $T_{1}, T_{2}, \ldots, T_{n}$ and $\mu_{1}, \mu_{2}, \ldots, \mu_{n}$.

For each $n, k \in \mathbb{N}$, let the mapping $U_{n, k}$ be defined by (2.11), then we can have the following crucial conclusions concerning $W_{n}$. You can find them in [23]. Now, we only need the following similar version in Hilbert spaces.

Lemma 2.2 (see [23]). Let $C$ be a nonempty closed-convex subset of a real Hilbert space H. Let $T_{1}, T_{2}, \ldots$ be nonexpansive mappings of $C$ into itself such that $\bigcap_{n=1}^{\infty} F\left(T_{n}\right)$ is nonempty, and let $\mu_{1}, \mu_{2}, \ldots$ be real numbers such that $0 \leq \mu_{n} \leq b<1$ for every $n \geq 1$, then

(1) $W_{n}$ is nonexpansive and $F\left(W_{n}\right)=\bigcap_{i=1}^{n} F\left(T_{i}\right), \forall n \geq 1$,

(2) for every $x \in C$ and $k \in \mathbb{N}$, the limit $\lim _{n \rightarrow \infty} U_{n, k} x$ exists,

(3) a mapping $W: C \rightarrow C$ defined by

$$
W x:=\lim _{n \rightarrow \infty} W_{n} x=\lim _{n \rightarrow \infty} U_{n, 1} x, \quad \forall x \in C
$$

is a nonexpansive mapping satisfying $F(W)=\bigcap_{i=1}^{\infty} F\left(T_{i}\right)$, and it is called the $W$-mapping generated by $T_{1}, T_{2}, \ldots$ and $\mu_{1}, \mu_{2}, \ldots$

Lemma 2.3 (see [24]). Let $C$ be a nonempty closed-convex subset of a Hilbert space $H$, let $\left\{T_{i}\right.$ : $C \rightarrow C\}$ be a countable family of nonexpansive mappings with $\bigcap_{i=1}^{\infty} F\left(T_{i}\right) \neq \emptyset$, and let $\left\{\mu_{i}\right\}$ be a real 
sequence such that $0<\mu_{i} \leq b<1, \forall i \geq 1$. If $D$ is any bounded subset of $C$, then

$$
\limsup _{n \rightarrow \infty} \sup _{x \in D} W x-W_{n} x \|=0
$$

Lemma 2.4 (see [25]). Each Hilbert space $H$ satisfies Opials condition, that is, for any sequence $\left\{x_{n}\right\} \subset H$ with $x_{n} \rightarrow x$, the inequality

$$
\liminf _{n \rightarrow \infty}\left\|x_{n}-x\right\|<\liminf _{n \rightarrow \infty}\left\|x_{n}-y\right\|
$$

holds for each $y \in H$ with $y \neq x$.

Lemma 2.5 (see [3]). Let $M: H \rightarrow 2^{H}$ be a maximal monotone mapping, and let $A: H \rightarrow H$ be a monotone mapping, then the mapping $S=M+A: H \rightarrow 2^{H}$ is a maximal monotone mapping.

Remark 2.6. Lemma 2.5 implies that $I(A, M)$ is closed and convex if $M: H \rightarrow 2^{H}$ is a maximal monotone mapping and $A: H \rightarrow H$ is a monotone mapping.

Lemma 2.7 (see [4]). Let $u \in H$ be a solution of variational inclusion (1.4) if and only if $u=$ $J_{M, \lambda}(u-\lambda A u), \forall \lambda>0$, that is,

$$
I(A, M)=F\left(J_{M, \lambda}(I-\lambda A)\right), \quad \forall \lambda>0 .
$$

Lemma 2.8 (see [20]). Let $C$ be a nonempty bounded closed-convex subset of a Hilbert space $H$, and let $\mathcal{S}=\{S(s): 0 \leq s<\infty\}$ be a nonexpansive semigroup on $C$, then for any $h \geq 0$,

$$
\limsup _{t \rightarrow \infty}\left\|\frac{1}{t} \int_{0}^{t} S(s) x d s-S(h)\left(\frac{1}{t} \int_{0}^{t} S(s) x d s\right)\right\|=0 .
$$

Lemma 2.9 (see [26]). Let $C$ be a nonempty bounded closed-convex subset of $H$, let $\left\{x_{n}\right\}$ be a sequence in $C$, and let $S=\{S(s): 0 \leq s<\infty\}$ be a nonexpansive semigroup on $C$. If the following conditions are satisfied:

(1) $x_{n} \rightarrow z$,

(2) $\limsup _{s \rightarrow \infty} \limsup _{n \rightarrow \infty}\left\|S(s) x_{n}-x_{n}\right\|=0$, then $z \in F(S)$.

For solving the generalized mixed equilibrium problem for $F: C \times C \rightarrow \mathbb{R}$, one gives the following assumptions for the bifunction $F, \varphi$ and the set $C$ :

(A1) $F(x, x)=0$ for all $x \in C$,

(A2) $F$ is monotone, that is, $F(x, y)+F(y, x) \leq 0$ for all $x, y \in C$,

(A3) for each $x, y, z \in C, \lim _{t \rightarrow 0} F(t z+(1-t) x, y) \leq F(x, y)$,

(A4) for each $x \in C, y \mapsto F(x, y)$ is convex and lower semicontinuous,

(A5) for each $y \in C, x \mapsto F(x, y)$ is weakly upper semicontinuous, 
(B1) for each $x \in H$ and $r>0$, there exist a bounded subset $D_{x} \subseteq C$ and $y_{x} \in C$ such that for any $z \in C \backslash D_{x}$,

$$
F\left(z, y_{x}\right)+\varphi\left(y_{x}\right)+\frac{1}{r}\left\langle y_{x}-z, z-x\right\rangle<\varphi(z)
$$

(B2) $C$ is a bounded set,

then one has the following lemma.

Lemma 2.10 (see [18]). Let $C$ be a nonempty closed-convex subset of $H$. Let $F: C \times C \rightarrow \mathbb{R}$ be a bifunction that satisfies (A1)-(A5), and let $\varphi: C \rightarrow \mathbb{R} \cup\{+\infty\}$ be a proper lower semicontinuous and convex function. Assume that either (B1) or (B2) holds. For $r>0$ and $x \in H$, define a mapping $T_{r}: H \rightarrow C$ as follows:

$$
T_{r}^{(F, \varphi)}(x)=\left\{z \in C: F(z, y)+\varphi(y)+\frac{1}{r}\langle y-z, z-x\rangle \geq \varphi(z), \forall y \in C\right\},
$$

for all $z \in H$, then the following hold:

(1) for each $x \in H, T_{r}^{(F, \varphi)}(x) \neq \emptyset$,

(2) $T_{r}^{(F, \varphi)}$ is single valued,

(3) $T_{r}^{(F, \varphi)}$ is firmly nonexpansive, that is, for any $x, y \in H,\left\|T_{r}^{(F, \varphi)} x-T_{r}^{(F, \varphi)} y\right\|^{2} \leq\left\langle T_{r}^{(F, \varphi)} x-\right.$ $\left.T_{r}^{(F, \varphi)} y, x-y\right\rangle$,

(4) $F\left(T_{r}^{(F, \varphi)}\right)=\operatorname{MEP}(F, \varphi)$,

(5) $\operatorname{MEP}(F, \varphi)$ is closed and convex.

\section{Main Result}

In this section, we prove a strong convergence theorem for finding a common element of the set of solutions for a system of generalized mixed equilibrium problems, the set of common solutions of variational inclusion problems with set-valued maximal monotone mappings and inverse-strongly monotone mappings, the set of solutions of fixed points for nonexpansive semigroup mappings, and the set of common fixed points for an infinite family of strictly pseudocontractive mappings in a real Hilbert space.

Theorem 3.1. Let $C$ be a nonempty closed-convex subset of a real Hilbert Space $H$. Let $F_{1}, F_{2}$ be bifunctions of $C \times C$ into real numbers $\mathbb{R}$ satisfying (A1)-(A5), and let $\varphi_{1}, \varphi_{2}: C \rightarrow \mathbb{R} \cup\{+\infty\}$ be proper lower semicontinuous and convex functions with assumption (B1) or (B2). Let $A, B, E_{1}, E_{2}$ be $\alpha, \beta, \eta_{1}, \eta_{2}$-inverse-strongly monotone mappings of $C$ into $H$, respectively, and let $M_{1}, M_{2}: H \rightarrow$ $2^{H}$ be maximal monotone mappings. Let $S=\{S(s): 0 \leq s<\infty\}$ be a nonexpansive semigroup on $C$, and let $\left\{t_{n}\right\}$ be a positive real divergent sequence. Let $\left\{V_{i}: C \rightarrow C\right\}_{i=1}^{\infty}$ be a countable family of uniformly $k$-strict pseudocontractions, let $\left\{T_{i}: C \rightarrow C\right\}_{i=1}^{\infty}$ be a countable family of nonexpansive mappings defined by $T_{i} x=t x+(1-t) V_{i} x, \forall x \in C, \forall i \geq 1, t \in[k, 1)$, and let $W_{n}$ be the $W$-mapping defined by (2.11) and $W$ a mapping defined by (2.12) with $F(W) \neq \emptyset$. Suppose that 
$\Theta:=F(S) \cap F(W) \cap G M E P\left(F_{1}, \varphi_{1}, A\right) \cap G M E P\left(F_{2}, \varphi_{2}, B\right) \cap I\left(E_{1}, M_{1}\right) \cap I\left(E_{2}, M_{2}\right) \neq \emptyset$. Let $\left\{x_{n}\right\}$ be a sequence generated by $x_{0} \in C, C_{1, i}=C, C_{1}=\bigcap_{i=1}^{\infty} C_{1, i}, x_{1}=P_{C_{1}} x_{0}$, and

$$
\begin{gathered}
t_{n}=T_{r_{n}}^{\left(F_{1}, \varphi_{1}\right)}\left(x_{n}-r_{n} A x_{n}\right), \\
u_{n}=T_{q_{n}}^{\left(F_{2,}, \varphi_{2}\right)}\left(t_{n}-q_{n} B t_{n}\right), \\
v_{n}=J_{M_{1}, \lambda_{1}}\left(u_{n}-\lambda_{1} E_{1} u_{n}\right), \\
w_{n}=J_{M_{2,}, \lambda_{2}}\left(v_{n}-\lambda_{2} E_{2} v_{n}\right), \\
C_{n+1, i}=\left\{z \in C_{n, i}:\left\|y_{n, i}-z\right\|^{2} \leq\left\|x_{n}-z\right\|^{2}+\alpha_{n, i}\left(\left\|x_{0}\right\|^{2}+2\left\langle x_{n}-x_{0}, z\right\rangle\right)\right\}, \\
C_{n+1}=\alpha_{n, i} x_{0}+\left(1-\alpha_{n, i}\right) \frac{1}{t_{n}} \int_{0}^{\infty} C_{n+1, i}, \\
x_{n+1}=P_{C_{n+1}} x_{0},
\end{gathered}
$$

for every $n \geq 0$, where $\left\{\alpha_{n, i}\right\}_{n=1}^{\infty} \subset(0,1),\left\{r_{n}\right\},\left\{q_{n}\right\} \subset(0, \infty), \lambda_{1} \in\left(0,2 \eta_{1}\right)$, and $\lambda_{2} \in\left(0,2 \eta_{2}\right)$ satisfy the following conditions:

(i) $0<a \leq r_{n} \leq b<2 \alpha$,

(ii) $0<c \leq q_{n} \leq d<2 \beta$,

(iii) $\lim _{n \rightarrow \infty} \alpha_{n, i}=0$,

(iv) $0<e \leq \lambda_{1} \leq f<2 \eta_{1}$,

(v) $0<g \leq \lambda_{2} \leq j<2 \eta_{2}$,

then $\left\{x_{n}\right\}$ converges strongly to $P_{\Theta} x_{0}$.

Proof. First, we show that $I-\lambda_{1} E_{1}$ and $I-\lambda_{2} E_{2}$ are nonexpansive. Indeed, for all $x, y \in C$ and $\lambda_{1} \in\left(0,2 \eta_{1}\right)$, we obtain

$$
\begin{aligned}
\left\|\left(I-\lambda_{1} E_{1}\right) x-\left(I-\lambda_{1} E_{1}\right) y\right\|^{2} & =\left\|x-y-\lambda_{1}\left(E_{1} x-E_{1} y\right)\right\|^{2} \\
& =\|x-y\|^{2}-2 \lambda_{1}\left\langle x-y, E_{1} x-E_{1} y\right\rangle+\lambda_{1}^{2}\left\|E_{1} x-E_{1} y\right\|^{2} \\
& \leq\|x-y\|^{2}-2 \lambda_{1} \eta_{1}\left\|E_{1} x-E_{1} y\right\|^{2}+\lambda_{1}^{2}\left\|E_{1} x-E_{1} y\right\|^{2} \\
& \leq\|x-y\|^{2}+\lambda_{1}\left(\lambda_{1}-2 \eta_{1}\right)\left\|E_{1} x-E_{1} y\right\|^{2} \\
& \leq\|x-y\|^{2},
\end{aligned}
$$


which implies that the mapping $I-\lambda_{1} E_{1}$ is nonexpansive, so is $I-\lambda_{2} E_{2}$. Let $p \in \Theta$. We observe that

$$
\begin{aligned}
\left\|w_{n}-p\right\|^{2} & =\left\|J_{M_{2}, \lambda_{2}}\left(v_{n}-\lambda_{2} E_{2} v_{n}\right)-J_{M_{2}, \lambda_{2}}\left(p-\lambda_{2} E_{2} p\right)\right\|^{2} \\
& \leq\left\|\left(v_{n}-\lambda_{2} E_{2} v_{n}\right)-\left(p-\lambda_{2} E_{2} p\right)\right\|^{2} \\
& \leq\left\|v_{n}-p\right\|^{2} \\
& =\left\|J_{M_{1}, \lambda_{1}}\left(u_{n}-\lambda_{1} E_{1} u_{n}\right)-J_{M_{1}, \lambda_{1}}\left(p-\lambda_{1} E_{1} p\right)\right\|^{2} \\
& \leq\left\|\left(u_{n}-\lambda_{1} E_{1} u_{n}\right)-\left(p-\lambda_{1} E_{1} p\right)\right\|^{2} \\
& =\left\|u_{n}-p\right\|^{2} .
\end{aligned}
$$

Since both $I-r_{n} A$ and $I-q_{n} B$ are nonexpansive for each $n \geq 1$, let $p \in \Theta$, then $p=T_{r_{n}}^{F_{1}, \varphi_{1}}(p-$ $\left.r_{n} A p\right)$ and $p=T_{q_{n}}^{F_{2}, \varphi_{2}}\left(p-q_{n} B p\right)$; by conditions (i) and (ii), we have

$$
\begin{aligned}
\left\|u_{n}-p\right\|^{2} & =\left\|T_{q_{n}}^{\left(F_{2}, \varphi_{2}\right)}\left(I-q_{n} B\right) t_{n}-T_{q_{n}}^{\left(F_{2}, \varphi_{2}\right)}\left(I-q_{n} B\right) p\right\|^{2} \\
& \leq\left\|\left(I-q_{n} B\right) t_{n}-\left(I-q_{n} B\right) p\right\|^{2} \\
& \leq\left\|t_{n}-p\right\|^{2}+q_{n}\left(q_{n}-2 \beta\right)\left\|B t_{n}-B p\right\|^{2} \\
& \leq\left\|t_{n}-p\right\|^{2}, \\
\left\|t_{n}-p\right\|^{2} & =\left\|T_{r_{n}}^{\left(F_{1}, \varphi_{1}\right)}\left(I-r_{n} A\right) x_{n}-T_{r_{n}}^{\left(F_{1}, \varphi_{1}\right)}\left(I-r_{n} A\right) p\right\|^{2} \\
& \leq\left\|\left(I-r_{n} A\right) x_{n}-\left(I-r_{n} A\right) p\right\|^{2} \\
& \leq\left\|x_{n}-p\right\|^{2}+r_{n}\left(r_{n}-2 \alpha\right)\left\|A x_{n}-A p\right\|^{2} \\
& \leq\left\|x_{n}-p\right\|^{2} .
\end{aligned}
$$

Therefore, we get

$$
\left\|u_{n}-p\right\| \leq\left\|x_{n}-p\right\|
$$

Next, we will divide the proof into five steps.

Step 1. We show that $\left\{x_{n}\right\}$ is well defined. Let $n=1$, then $C_{1, i}=C$ is closed and convex for each $i \geq 1$. Suppose that $C_{n, i}$ is closed convex for some $n>1$, then, from the definition of $C_{n+1, i}$, we know that $C_{n+1, i}$ is closed convex for the same $n \geq 1$. Hence, $C_{n, i}$ is closed convex 
for $n \geq 1$ and for each $i \geq 1$. This implies that $C_{n}$ is closed convex for $n \geq 1$. Furthermore, we show that $\Theta \subset C_{n}$. For $n=1, \Theta \subset C=C_{1, i}$. For $n \geq 2$, let $p \in \Theta$, then

$$
\begin{aligned}
\left\|y_{n, i}-p\right\|^{2} & =\left\|\alpha_{n, i}\left(x_{0}-p\right)+\left(1-\alpha_{n, i}\right)\left(\frac{1}{t_{n}} \int_{0}^{t_{n}} S(s) W_{n} w_{n} d s-p\right)\right\|^{2} \\
& \leq \alpha_{n, i}\left\|x_{0}-p\right\|^{2}+\left(1-\alpha_{n, i}\right)\left\|\frac{1}{t_{n}} \int_{0}^{t_{n}} S(s) W_{n} w_{n} d s-p\right\|^{2} \\
& \leq \alpha_{n, i}\left\|x_{0}-p\right\|^{2}+\left(1-\alpha_{n, i}\right)\left\|w_{n}-p\right\|^{2} \\
& =\left\|w_{n}-p\right\|^{2}+\alpha_{n, i}\left(\left\|x_{0}-p\right\|^{2}-\left\|w_{n}-p\right\|^{2}\right) \\
& \leq\left\|x_{n}-p\right\|^{2}+\alpha_{n, i}\left(\left\|x_{0}\right\|^{2}+2\left\langle x_{n}-x_{0}, p\right\rangle\right)
\end{aligned}
$$

which shows that $p \in C_{n, i}, \forall n \geq 2, \forall i \geq 1$. Thus, $\Theta \subset C_{n, i}, \forall n \geq 1, \forall i \geq 1$. Hence, it follows that $\emptyset \neq \Theta \subset C_{n}, \forall n \geq 1$. This implies that $\left\{x_{n}\right\}$ is well defined.

Step 2. We claim that $\lim _{n \rightarrow \infty}\left\|x_{n+1}-x_{n}\right\|=0$ and $\lim _{n \rightarrow \infty}\left\|y_{n, i}-x_{n}\right\|=0$, for $i \geq 1$. Since $x_{n}=P_{C_{n}} x_{0}$ and $x_{n+1}=P_{C_{n+1}} x_{0} \in C_{n+1} \subset C_{n}, \forall n \geq 1$, we have

$$
\left\|x_{n}-x_{0}\right\| \leq\left\|x_{n+1}-x_{0}\right\|, \quad \forall n \geq 1
$$

Also, as $\Theta \subset C_{n}$ by (2.1), it follows that

$$
\left\|x_{n}-x_{0}\right\| \leq\left\|z-x_{0}\right\|, \quad z \in \Theta, \forall n \geq 1 .
$$

Form (3.7) and (3.8), we have that $\lim _{n \rightarrow \infty}\left\|x_{n}-x_{0}\right\|$ exists. Hence, $\left\{x_{n}\right\}$ is bounded and so are $\left\{y_{n, i}\right\}, \forall i \geq 1,\left\{w_{n}\right\},\left\{v_{n}\right\},\left\{u_{n}\right\},\left\{t_{n}\right\},\left\{A x_{n}\right\},\left\{B t_{n}\right\},\left\{E_{1} u_{n}\right\},\left\{E_{2} v_{n}\right\},\left\{W_{n} w_{n}\right\}$, and $\left\{\left(1 / t_{n}\right) \int_{0}^{t_{n}} S(s) W_{n} w_{n} d s\right\}$. For $m>n \geq 1$, we have that $x_{m}=P_{C_{m}} x_{0} \in C_{m} \subset C_{n}$. By (2.5), we obtain

$$
\left\|x_{m}-x_{n}\right\|^{2} \leq\left\|x_{n}-x_{0}\right\|^{2}-\left\|x_{m}-x_{0}\right\|^{2}
$$

Letting $m, n \rightarrow \infty$ and taking the limit in (3.9), we have $\left\|x_{m}-x_{n}\right\| \rightarrow 0$, which shows that $\left\{x_{n}\right\}$ is Cauchy. In particular,

$$
\lim _{n \rightarrow \infty}\left\|x_{n+1}-x_{n}\right\|=0 .
$$


Since $\left\{x_{n}\right\}$ is Cauchy, we assume that $x_{n} \rightarrow z \in C$. Since $x_{n+1}=P_{C_{n+1}} x_{0} \in C_{n+1}$, then

$$
\left\|y_{n, i}-x_{n+1}\right\|^{2} \leq\left\|x_{n}-x_{n+1}\right\|^{2}+\alpha_{n, i}\left(\left\|x_{0}\right\|^{2}+2\left\langle x_{n}-x_{0}, x_{n+1}\right\rangle\right) \longrightarrow 0,
$$

and it follows that

$$
\left\|y_{n, i}-x_{n}\right\| \leq\left\|y_{n, i}-x_{n+1}\right\|+\left\|x_{n+1}-x_{n}\right\|
$$

Therefore,

$$
\lim _{n \rightarrow \infty}\left\|y_{n, i}-x_{n}\right\|=0, \quad \forall i \geq 1
$$

Step 3. We claim that the following statements hold:

(1) $\lim _{n \rightarrow \infty}\left\|u_{n}-t_{n}\right\|=0$,

(2) $\lim _{n \rightarrow \infty}\left\|t_{n}-x_{n}\right\|=0$,

(3) $\lim _{n \rightarrow \infty}\left\|w_{n}-v_{n}\right\|=0$,

(4) $\lim _{n \rightarrow \infty}\left\|v_{n}-u_{n}\right\|=0$.

For $p \in \Theta$, from (3.4), and (3.6), we obtain

$$
\begin{aligned}
\left\|y_{n, i}-p\right\|^{2} & \leq \alpha_{n, i}\left\|x_{0}-p\right\|^{2}+\left(1-\alpha_{n, i}\right)\left\|w_{n}-p\right\|^{2} \\
& \leq \alpha_{n, i}\left\|x_{0}-p\right\|^{2}+\left(1-\alpha_{n, i}\right)\left\|u_{n}-p\right\|^{2} \\
& \leq \alpha_{n, i}\left\|x_{0}-p\right\|^{2}+\left(1-\alpha_{n, i}\right)\left(\left\|t_{n}-p\right\|^{2}+q_{n}\left(q_{n}-2 \beta\right)\left\|B t_{n}-B p\right\|^{2}\right) \\
& \leq \alpha_{n, i}\left\|x_{0}-p\right\|^{2}+\left\|x_{n}-p\right\|^{2}+\left(1-\alpha_{n, i}\right) q_{n}\left(q_{n}-2 \beta\right)\left\|B t_{n}-B p\right\|^{2} .
\end{aligned}
$$

Since $0<c \leq q_{n} \leq d<2 \beta$, we have

$$
\begin{aligned}
\left(1-\alpha_{n, i}\right) q_{n}\left(2 \beta-q_{n}\right)\left\|B t_{n}-B p\right\|^{2} & \leq \alpha_{n, i}\left\|x_{0}-p\right\|^{2}+\left\|x_{n}-p\right\|^{2}-\left\|y_{n, i}-p\right\|^{2} \\
& \leq \alpha_{n, i}\left\|x_{0}-p\right\|^{2}+\left\|x_{n}-y_{n, i}\right\|\left(\left\|x_{n}-p\right\|+\left\|y_{n, i}-p\right\|\right) .
\end{aligned}
$$

Hence, by condition (iii) and (3.13), we have

$$
\lim _{n \rightarrow \infty}\left\|B t_{n}-B p\right\|=0 .
$$


From (3.6), we have

$$
\begin{aligned}
\left\|y_{n, i}-p\right\|^{2} & \leq \alpha_{n, i}\left\|x_{0}-p\right\|^{2}+\left(1-\alpha_{n, i}\right)\left\|w_{n}-p\right\|^{2} \\
& \leq \alpha_{n, i}\left\|x_{0}-p\right\|^{2}+\left(1-\alpha_{n, i}\right)\left\|u_{n}-p\right\|^{2} .
\end{aligned}
$$

On the other hand,

$$
\begin{aligned}
\left\|u_{n}-p\right\|^{2}= & \left\|T_{q_{n}}^{\left(F_{2}, \varphi_{2}\right)}\left(t_{n}-q_{n} B t_{n}\right)-T_{q_{n}}^{\left(F_{2}, \varphi_{2}\right)}\left(p-q_{n} B p\right)\right\|^{2} \\
\leq & \left\langle\left(t_{n}-q_{n} B t_{n}\right)-\left(p-q_{n} B p\right), u_{n}-p\right\rangle \\
= & \frac{1}{2}\left\{\left\|\left(t_{n}-q_{n} B t_{n}\right)-\left(p-q_{n} B p\right)\right\|^{2}+\left\|u_{n}-p\right\|^{2}\right. \\
& \left.\quad-\left\|\left(t_{n}-q_{n} B t_{n}\right)-\left(p-q_{n} B p\right)-\left(u_{n}-p\right)\right\|^{2}\right\} \\
\leq & \frac{1}{2}\left\{\left\|t_{n}-p\right\|^{2}+\left\|u_{n}-p\right\|^{2}-\left\|\left(t_{n}-q_{n} B t_{n}\right)-\left(p-q_{n} B p\right)-\left(u_{n}-p\right)\right\|^{2}\right\} \\
\leq & \frac{1}{2}\left\{\left\|x_{n}-p\right\|^{2}+\left\|u_{n}-p\right\|^{2}-\left\|u_{n}-t_{n}\right\|^{2}+2 q_{n}\left\langle t_{n}-u_{n}, B t_{n}-B p\right\rangle-s_{n}^{2}\left\|B t_{n}-B p\right\|^{2}\right\},
\end{aligned}
$$

and hence,

$$
\left\|u_{n}-p\right\|^{2} \leq\left\|x_{n}-p\right\|^{2}-\left\|u_{n}-t_{n}\right\|^{2}+2 q_{n}\left\|t_{n}-u_{n}\right\|\left\|B t_{n}-B p\right\| .
$$

Putting (3.19) into (3.17), for $i \geq 1$, we have

$$
\begin{aligned}
\left\|y_{n, i}-p\right\|^{2} & \leq \alpha_{n, i}\left\|x_{0}-p\right\|^{2}+\left(1-\alpha_{n, i}\right)\left(\left\|x_{n}-p\right\|^{2}-\left\|u_{n}-t_{n}\right\|^{2}+2 q_{n}\left\|t_{n}-u_{n}\right\|\left\|B t_{n}-B p\right\|\right) \\
& \leq \alpha_{n, i}\left\|x_{0}-p\right\|^{2}+\left\|x_{n}-p\right\|^{2}-\left(1-\alpha_{n, i}\right)\left\|u_{n}-t_{n}\right\|^{2}+2 q_{n}\left\|t_{n}-u_{n}\right\|\left\|B t_{n}-B p\right\| .
\end{aligned}
$$

It follows that

$$
\begin{aligned}
\left(1-\alpha_{n, i}\right)\left\|u_{n}-t_{n}\right\|^{2} \leq & \alpha_{n, i}\left\|x_{0}-p\right\|^{2}+\left\|x_{n}-p\right\|^{2}-\left\|y_{n, i}-p\right\|^{2}+2 q_{n}\left\|t_{n}-u_{n}\right\|\left\|B t_{n}-B p\right\| \\
\leq & \alpha_{n, i}\left\|x_{0}-p\right\|^{2}+\left\|x_{n}-y_{n, i}\right\|\left(\left\|x_{n}-p\right\|+\left\|y_{n, i}-p\right\|\right) \\
& +2 q_{n}\left\|t_{n}-u_{n}\right\|\left\|B t_{n}-B p\right\| .
\end{aligned}
$$


Therefore, from condition (iii), (3.13), and (3.16), we have

$$
\lim _{n \rightarrow \infty}\left\|u_{n}-t_{n}\right\|=0 .
$$

Furthermore, from (3.4), and (3.6), we get

$$
\begin{aligned}
\left\|y_{n, i}-p\right\|^{2} & \leq \alpha_{n, i}\left\|x_{0}-p\right\|^{2}+\left(1-\alpha_{n, i}\right)\left\|w_{n}-p\right\|^{2} \\
& \leq \alpha_{n, i}\left\|x_{0}-p\right\|^{2}+\left(1-\alpha_{n, i}\right)\left\|u_{n}-p\right\|^{2} \\
& \leq \alpha_{n, i}\left\|x_{0}-p\right\|^{2}+\left(1-\alpha_{n, i}\right)\left\|t_{n}-p\right\|^{2} \\
& \leq \alpha_{n, i}\left\|x_{0}-p\right\|^{2}+\left(1-\alpha_{n, i}\right)\left\{\left\|x_{n}-p\right\|^{2}+r_{n}\left(r_{n}-2 \alpha\right)\left\|A x_{n}-A p\right\|^{2}\right\} \\
& \leq \alpha_{n, i}\left\|x_{0}-p\right\|^{2}+\left\|x_{n}-p\right\|^{2}+\left(1-\alpha_{n, i}\right) r_{n}\left(r_{n}-2 \alpha\right)\left\|A x_{n}-A p\right\|^{2} .
\end{aligned}
$$

Since $0<a \leq r_{n} \leq b<2 \alpha$, we have

$$
\begin{aligned}
\left(1-\alpha_{n, i}\right) r_{n}\left(2 \alpha-r_{n}\right)\left\|A x_{n}-A p\right\|^{2} & \leq \alpha_{n, i}\left\|x_{0}-p\right\|^{2}+\left\|x_{n}-p\right\|^{2}-\left\|y_{n, i}-p\right\|^{2} \\
& \leq \alpha_{n, i}\left\|x_{0}-p\right\|^{2}+\left\|x_{n}-y_{n, i}\right\|\left(\left\|x_{n}-p\right\|+\left\|y_{n, i}-p\right\|\right)
\end{aligned}
$$

Then, by condition (iii) and (3.13), we obtain that

$$
\lim _{n \rightarrow \infty}\left\|A x_{n}-A p\right\|=0 .
$$

From (3.6), we have

$$
\begin{aligned}
\left\|y_{n, i}-p\right\|^{2} & \leq \alpha_{n, i}\left\|x_{0}-p\right\|^{2}+\left(1-\alpha_{n, i}\right)\left\|w_{n}-p\right\|^{2} \\
& \leq \alpha_{n, i}\left\|x_{0}-p\right\|^{2}+\left(1-\alpha_{n, i}\right)\left\|u_{n}-p\right\|^{2} \\
& \leq \alpha_{n, i}\left\|x_{0}-p\right\|^{2}+\left(1-\alpha_{n, i}\right)\left\|t_{n}-p\right\|^{2} .
\end{aligned}
$$


On the other hand, we note that

$$
\begin{aligned}
\left\|t_{n}-p\right\|^{2} \leq & \left\|T_{r_{n}}^{\left(F_{1}, \varphi_{1}\right)}\left(x_{n}-r_{n} A x_{n}\right)-T_{r_{n}}^{\left(F_{1}, \varphi_{1}\right)}\left(p-r_{n} A p\right)\right\|^{2} \\
\leq & \left\langle\left(x_{n}-r_{n} A x_{n}\right)-\left(p-r_{n} A p\right), t_{n}-p\right\rangle \\
= & \frac{1}{2}\left\{\left\|\left(x_{n}-r_{n} A x_{n}\right)-\left(p-r_{n} A p\right)\right\|^{2}+\left\|t_{n}-p\right\|^{2}\right. \\
& \left.\quad-\left\|\left(x_{n}-r_{n} A x_{n}\right)-\left(p-r_{n} A p\right)-\left(t_{n}-p\right)\right\|^{2}\right\} \\
\leq & \frac{1}{2}\left\{\left\|x_{n}-p\right\|^{2}+\left\|t_{n}-p\right\|^{2}-\left\|\left(x_{n}-r_{n} A x_{n}\right)-\left(p-r_{n} A p\right)-\left(t_{n}-p\right)\right\|^{2}\right\} \\
= & \frac{1}{2}\left\{\left\|x_{n}-p\right\|^{2}+\left\|t_{n}-p\right\|^{2}-\left\|t_{n}-x_{n}\right\|^{2}+2 r_{n}\left\langle x_{n}-t_{n}, A x_{n}-A p\right\rangle-r_{n}^{2}\left\|A x_{n}-A p\right\|^{2}\right\},
\end{aligned}
$$

and hence,

$$
\left\|t_{n}-p\right\|^{2} \leq\left\|x_{n}-p\right\|^{2}-\left\|t_{n}-x_{n}\right\|^{2}+2 r_{n}\left\|x_{n}-t_{n}\right\|\left\|A x_{n}-A p\right\| .
$$

Putting (3.28) into (3.26), we have

$$
\begin{aligned}
\left\|y_{n, i}-p\right\|^{2} & \leq \alpha_{n, i}\left\|x_{0}-p\right\|^{2}+\left(1-\alpha_{n, i}\right)\left(\left\|x_{n}-p\right\|^{2}-\left\|t_{n}-x_{n}\right\|^{2}+2 r_{n}\left\|x_{n}-t_{n}\right\|\left\|A x_{n}-A p\right\|\right) \\
& \leq \alpha_{n, i}\left\|x_{0}-p\right\|^{2}+\left\|x_{n}-p\right\|^{2}-\left(1-\alpha_{n, i}\right)\left\|t_{n}-x_{n}\right\|^{2}+2 r_{n}\left\|x_{n}-t_{n}\right\|\left\|A x_{n}-A p\right\| .
\end{aligned}
$$

It follows that

$$
\begin{aligned}
\left(1-\alpha_{n, i}\right)\left\|x_{n}-t_{n}\right\|^{2} \leq & \alpha_{n, i}\left\|x_{0}-p\right\|^{2}+\left\|x_{n}-p\right\|^{2}-\left\|y_{n, i}-p\right\|^{2}+2 r_{n}\left\|x_{n}-z_{n}\right\|\left\|A x_{n}-A p\right\| \\
\leq & \alpha_{n, i}\left\|x_{0}-p\right\|^{2}+\left\|x_{n}-y_{n, i}\right\|\left(\left\|x_{n}-p\right\|+\left\|y_{n, i}-p\right\|\right) \\
& +2 r_{n}\left\|x_{n}-z_{n}\right\|\left\|A x_{n}-A p\right\| .
\end{aligned}
$$

Therefore, by condition (iii), (3.13), and (3.25), we have

$$
\lim _{n \rightarrow \infty}\left\|x_{n}-t_{n}\right\|=0
$$


Condition (iii) implies that

$$
\left\|y_{n, i}-\frac{1}{t_{n}} \int_{0}^{t_{n}} S(s) W_{n} w_{n} d s\right\|^{2}=\alpha_{n, i}\left\|x_{0}-\frac{1}{t_{n}} \int_{0}^{t_{n}} S(s) W_{n} w_{n} d s\right\| \longrightarrow 0 .
$$

It follows that

$$
\left\|x_{n}-\frac{1}{t_{n}} \int_{0}^{t_{n}} S(s) W_{n} w_{n} d s\right\| \leq\left\|x_{n}-y_{n, i}\right\|+\left\|y_{n, i}+\frac{1}{t_{n}} \int_{0}^{t_{n}} S(s) W_{n} w_{n} d s\right\| \longrightarrow 0 .
$$

From (3.6), we have

$$
\begin{aligned}
\left\|y_{n, i}-p\right\|^{2} & \leq \alpha_{n, i}\left\|x_{0}-p\right\|^{2}+\left(1-\alpha_{n, i}\right)\left\|w_{n}-p\right\|^{2} \\
& =\alpha_{n, i}\left\|x_{0}-p\right\|^{2}+\left(1-\alpha_{n, i}\right)\left\|J_{M_{2}, \lambda_{2}}\left(v_{n}-\lambda_{2} E_{2} v_{n}\right)-J_{M_{2}, \lambda_{2}}\left(p-\lambda_{2} E_{2} p\right)\right\|^{2} \\
& \leq \alpha_{n, i}\left\|x_{0}-p\right\|^{2}+\left(1-\alpha_{n, i}\right)\left\|\left(v_{n}-\lambda_{2} E_{2} v_{n}\right)-\left(p-\lambda_{2} E_{2} p\right)\right\|^{2} \\
& \leq \alpha_{n, i}\left\|x_{0}-p\right\|^{2}+\left(1-\alpha_{n, i}\right)\left(\left\|v_{n}-p\right\|^{2}+\lambda_{2}\left(\lambda_{2}-2 \eta_{2}\right)\left\|E_{2} v_{n}-E_{2} p\right\|^{2}\right) \\
& \leq \alpha_{n, i}\left\|x_{0}-p\right\|^{2}+\left\|x_{n}-p\right\|^{2}+\left(1-\alpha_{n, i}\right) \lambda_{2}\left(\lambda_{2}-2 \eta_{2}\right)\left\|E_{2} v_{n}-E_{2} p\right\|^{2} .
\end{aligned}
$$

Since $0<g \leq \lambda_{2} \leq j<2 \eta_{2}$, we have

$$
\begin{aligned}
\left(1-\alpha_{n, i}\right) \lambda_{2}\left(2 \eta_{2}-\lambda_{2}\right)\left\|E_{2} v_{n}-E_{2} p\right\|^{2} & \leq \alpha_{n, i}\left\|x_{0}-p\right\|^{2}+\left\|x_{n}-p\right\|^{2}-\left\|y_{n, i}-p\right\|^{2} \\
& \leq \alpha_{n, i}\left\|x_{0}-p\right\|^{2}+\left\|x_{n}-y_{n, i}\right\|\left(\left\|x_{n}-p\right\|+\left\|y_{n, i}-p\right\|\right)
\end{aligned}
$$

Then, by condition (iii) and (3.13), we obtain that

$$
\lim _{n \rightarrow \infty}\left\|E_{2} v_{n}-E_{2} p\right\|=0
$$


From (3.6), we have

$$
\left\|y_{n, i}-p\right\|^{2} \leq \alpha_{n, i}\left\|x_{0}-p\right\|^{2}+\left(1-\alpha_{n, i}\right)\left\|w_{n}-p\right\|^{2}
$$

On the other hand, we note that

$$
\begin{aligned}
\left\|w_{n}-p\right\|^{2} \leq & \left\|J_{M_{2}, \lambda_{2}}\left(v_{n}-\lambda_{2} E_{2} v_{n}\right)-J_{M_{2}, \lambda_{2}}\left(p-\lambda_{2} E_{2} p\right)\right\|^{2} \\
\leq & \left\langle\left(v_{n}-\lambda_{2} E_{2} v_{n}\right)-\left(p-\lambda_{2} E_{2} p\right), w_{n}-p\right\rangle \\
= & \frac{1}{2}\left\{\left\|\left(v_{n}-\lambda_{2} E_{2} v_{n}\right)-\left(p-\lambda_{2} E_{2} p\right)\right\|^{2}+\left\|w_{n}-p\right\|^{2}\right. \\
& \left.\quad-\left\|\left(v_{n}-\lambda_{2} E_{2} v_{n}\right)-\left(p-\lambda_{2} E_{2} p\right)-\left(w_{n}-p\right)\right\|^{2}\right\} \\
\leq & \frac{1}{2}\left\{\left\|v_{n}-p\right\|^{2}+\left\|w_{n}-p\right\|^{2}-\left\|\left(v_{n}-\lambda_{2} E_{2} v_{n}\right)-\left(p-\lambda_{2} E_{2} p\right)-\left(w_{n}-p\right)\right\|^{2}\right\} \\
\leq & \frac{1}{2}\left\{\left\|x_{n}-p\right\|^{2}+\left\|w_{n}-p\right\|^{2}-\left\|w_{n}-v_{n}\right\|^{2}+2 \lambda_{2}\left\langle v_{n}-w_{n}, E_{2} v_{n}-E_{2} p\right\rangle\right. \\
& \left.\quad-\lambda_{2}^{2}\left\|E_{2} v_{n}-E_{2} p\right\|^{2}\right\},
\end{aligned}
$$

and hence,

$$
\left\|w_{n}-p\right\|^{2} \leq\left\|x_{n}-p\right\|^{2}-\left\|w_{n}-v_{n}\right\|^{2}+2 \lambda_{2}\left\|v_{n}-w_{n}\right\|\left\|E_{2} v_{n}-E_{2} p\right\| .
$$

Putting (3.39) into (3.37),

$$
\begin{aligned}
\left\|y_{n, i}-p\right\|^{2} & \leq \alpha_{n, i}\left\|x_{0}-p\right\|^{2}+\left(1-\alpha_{n, i}\right)\left(\left\|x_{n}-p\right\|^{2}-\left\|w_{n}-v_{n}\right\|^{2}+2 \lambda_{2}\left\|v_{n}-w_{n}\right\|\left\|E_{2} v_{n}-E_{2} p\right\|\right) \\
& \leq \alpha_{n, i}\left\|x_{0}-p\right\|^{2}+\left\|x_{n}-p\right\|^{2}-\left(1-\alpha_{n, i}\right)\left\|w_{n}-v_{n}\right\|^{2}+2 \lambda_{2}\left\|v_{n}-w_{n}\right\|\left\|E_{2} v_{n}-E_{2} p\right\|
\end{aligned}
$$

this implies that

$$
\begin{gathered}
\left(1-\alpha_{n, i}\right)\left\|w_{n}-v_{n}\right\|^{2} \leq \alpha_{n, i}\left\|x_{0}-p\right\|^{2}+\left\|x_{n}-p\right\|^{2}-\left\|y_{n, i}-p\right\|^{2}+2 \lambda_{2}\left\|w_{n}-v_{n}\right\|\left\|E_{2} v_{n}-E_{2} p\right\| \\
\leq \alpha_{n, i}\left\|x_{0}-p\right\|^{2}+\left\|x_{n}-y_{n, i}\right\|\left(\left\|x_{n}-p\right\|+\left\|y_{n, i}-p\right\|\right) \\
\quad+2 \lambda_{2}\left\|w_{n}-v_{n}\right\|\left\|E_{2} v_{n}-E_{2} p\right\| .
\end{gathered}
$$


Therefore, by condition (iii), (3.13), and (3.36), we have

$$
\lim _{n \rightarrow \infty}\left\|w_{n}-v_{n}\right\|=0
$$

Furthermore, from (3.6), we have

$$
\begin{aligned}
\left\|y_{n, i}-p\right\|^{2} & \leq \alpha_{n, i}\left\|x_{0}-p\right\|^{2}+\left(1-\alpha_{n, i}\right)\left\|w_{n}-p\right\|^{2} \\
& \leq \alpha_{n, i}\left\|x_{0}-p\right\|^{2}+\left(1-\alpha_{n, i}\right)\left\|v_{n}-p\right\|^{2} \\
& =\alpha_{n, i}\left\|x_{0}-p\right\|^{2}+\left(1-\alpha_{n, i}\right)\left\|J_{M_{1}, \lambda_{1}}\left(u_{n}-\lambda_{1} E_{1} u_{n}\right)-J_{M_{1}, \lambda_{1}}\left(p-\lambda_{1} E_{1} p\right)\right\|^{2} \\
& \leq \alpha_{n, i}\left\|x_{0}-p\right\|^{2}+\left(1-\alpha_{n, i}\right)\left\|\left(u_{n}-\lambda_{1} E_{1} u_{n}\right)-\left(p-\lambda_{1} E_{1} p\right)\right\|^{2} \\
& \leq \alpha_{n, i}\left\|x_{0}-p\right\|^{2}+\left(1-\alpha_{n, i}\right)\left(\left\|u_{n}-p\right\|^{2}+\lambda_{1}\left(\lambda_{1}-2 \eta_{1}\right)\left\|E_{1} u_{n}-E_{1} p\right\|^{2}\right) \\
& \leq \alpha_{n, i}\left\|x_{0}-p\right\|^{2}+\left\|x_{n}-p\right\|^{2}+\left(1-\alpha_{n, i}\right) \lambda_{1}\left(\lambda_{1}-2 \eta_{1}\right)\left\|E_{1} u_{n}-E_{1} p\right\|^{2} .
\end{aligned}
$$

Since $0<e \leq \lambda_{1} \leq f<2 \eta_{1}$, we have

$$
\begin{aligned}
& \left(1-\alpha_{n, i}\right) \lambda_{1}\left(2 \eta_{1}-\lambda_{1}\right)\left\|E_{1} u_{n}-E_{1} p\right\|^{2} \\
& \quad \leq \alpha_{n, i}\left\|x_{0}-p\right\|^{2}+\left\|x_{n}-p\right\|^{2}-\left\|y_{n, i}-p\right\|^{2} \\
& \quad \leq \alpha_{n, i}\left\|x_{0}-p\right\|^{2}+\left\|x_{n}-y_{n, i}\right\|\left(\left\|x_{n}-p\right\|+\left\|y_{n, i}-p\right\|\right) .
\end{aligned}
$$

Then, by condition (iii) and (3.13), we obtain that

$$
\lim _{n \rightarrow \infty}\left\|E_{1} u_{n}-E_{1} p\right\|=0
$$

From (3.6), we have

$$
\begin{aligned}
\left\|y_{n, i}-p\right\|^{2} & \leq \alpha_{n, i}\left\|x_{0}-p\right\|^{2}+\left(1-\alpha_{n, i}\right)\left\|w_{n}-p\right\|^{2} \\
& \leq \alpha_{n, i}\left\|x_{0}-p\right\|^{2}+\left(1-\alpha_{n, i}\right)\left\|v_{n}-p\right\|^{2} .
\end{aligned}
$$


Journal of Applied Mathematics

On the other hand, we note that

$$
\begin{aligned}
\left\|v_{n}-p\right\|^{2} \leq & \left\|J_{M_{1}, \lambda_{1}}\left(u_{n}-\lambda_{1} E_{1} u_{n}\right)-J_{M_{1}, \lambda_{1}}\left(p-\lambda_{1} E_{1} p\right)\right\|^{2} \\
\leq & \left\langle\left(u_{n}-\lambda_{1} E_{1} u_{n}\right)-\left(p-\lambda_{1} E_{1} p\right), v_{n}-p\right\rangle \\
= & \frac{1}{2}\left\{\left\|\left(u_{n}-\lambda_{1} E_{1} u_{n}\right)-\left(p-\lambda_{1} E_{1} p\right)\right\|^{2}+\left\|v_{n}-p\right\|^{2}\right. \\
& \left.\quad-\left\|\left(u_{n}-\lambda_{1} E_{1} u_{n}\right)-\left(p-\lambda_{1} E_{1} p\right)-\left(v_{n}-p\right)\right\|^{2}\right\} \\
\leq & \frac{1}{2}\left\{\left\|u_{n}-p\right\|^{2}+\left\|v_{n}-p\right\|^{2}-\left\|\left(u_{n}-\lambda_{1} E_{1} u_{n}\right)-\left(p-\lambda_{1} E_{1} p\right)-\left(v_{n}-p\right)\right\|^{2}\right\} \\
\leq & \frac{1}{2}\left\{\left\|x_{n}-p\right\|^{2}+\left\|v_{n}-p\right\|^{2}-\left\|v_{n}-u_{n}\right\|^{2}+2 \lambda_{1}\left\langle u_{n}-v_{n}, E_{1} u_{n}-E_{1} p\right\rangle\right. \\
& \left.\quad-\lambda_{1}^{2}\left\|E_{1} u_{n}-E_{1} p\right\|^{2}\right\},
\end{aligned}
$$

and hence,

$$
\left\|v_{n}-p\right\|^{2} \leq\left\|x_{n}-p\right\|^{2}-\left\|v_{n}-u_{n}\right\|^{2}+2 \lambda_{1}\left\|u_{n}-v_{n}\right\|\left\|E_{1} u_{n}-E_{1} p\right\| .
$$

Putting (3.48) into (3.46),

$$
\begin{aligned}
\left\|y_{n, i}-p\right\|^{2} & \leq \alpha_{n, i}\left\|x_{0}-p\right\|^{2}+\left(1-\alpha_{n, i}\right)\left(\left\|x_{n}-p\right\|^{2}-\left\|v_{n}-u_{n}\right\|^{2}+2 \lambda_{1}\left\|u_{n}-v_{n}\right\|\left\|E_{1} u_{n}-E_{1} p\right\|\right) \\
& \leq \alpha_{n, i}\left\|x_{0}-p\right\|^{2}+\left\|x_{n}-p\right\|^{2}-\left(1-\alpha_{n, i}\right)\left\|v_{n}-u_{n}\right\|^{2}+2 \lambda_{1}\left\|u_{n}-v_{n}\right\|\left\|E_{1} u_{n}-E_{1} p\right\|,
\end{aligned}
$$

this implies that

$$
\begin{aligned}
\left(1-\alpha_{n, i}\right)\left\|v_{n}-u_{n}\right\|^{2} \leq & \alpha_{n, i}\left\|x_{0}-p\right\|^{2}+\left\|x_{n}-p\right\|^{2}-\left\|y_{n, i}-p\right\|^{2}+2 \lambda_{1}\left\|v_{n}-u_{n}\right\|\left\|E_{1} u_{n}-E_{1} p\right\| \\
\leq & \alpha_{n, i}\left\|x_{0}-p\right\|^{2}+\left\|x_{n}-y_{n, i}\right\|\left(\left\|x_{n}-p\right\|+\left\|y_{n, i}-p\right\|\right) \\
& +2 \lambda_{1}\left\|v_{n}-u_{n}\right\|\left\|E_{1} u_{n}-E_{1} p\right\| .
\end{aligned}
$$

Therefore, by condition (iii), (3.13), and (3.45), we have

$$
\lim _{n \rightarrow \infty}\left\|v_{n}-u_{n}\right\|=0
$$

Step 4. We show that $z \in \Theta:=F(S) \cap F(W) \cap \operatorname{GMEP}\left(F_{1}, \varphi_{1}, A\right) \cap \operatorname{GMEP}\left(F_{2}, \varphi_{2}, B\right) \cap I\left(E_{1}, M_{1}\right) \cap$ $I\left(E_{2}, M_{2}\right)$. Since $\left\{w_{n_{i}}\right\}$ is bounded, there exists a subsequence $\left\{w_{n_{i_{j}}}\right\}$ of $\left\{w_{n_{i}}\right\}$ which converges weakly to $z \in C$. Without loss of generality, we can assume that $w_{n_{i}} \rightarrow z$. 
(1) First, we prove that $z \in F(\mathcal{S})$. From (3.22), (3.31), (3.33), (3.42), and (3.51), we get

$$
\lim _{n \rightarrow \infty}\left\|w_{n}-\frac{1}{t_{n}} \int_{0}^{t_{n}} S(s) W_{n} w_{n} d s\right\|=0
$$

Since $\left\{W_{n} w_{n}\right\}$ is bounded and from Lemma 2.8 for all $\mathrm{s} \geq 0$, we have

$$
\lim _{n \rightarrow \infty}\left\|\frac{1}{t_{n}} \int_{0}^{t_{n}} S(s) W_{n} w_{n} d s-S(s)\left(\frac{1}{t_{n}} \int_{0}^{t_{n}} S(s) W_{n} w_{n} d s\right)\right\|=0,
$$

and since

$$
\begin{aligned}
\left\|w_{n}-S(s) w_{n}\right\| \leq & \left\|w_{n}-\frac{1}{t_{n}} \int_{0}^{t_{n}} S(s) W_{n} w_{n} d s\right\| \\
& +\left\|\frac{1}{t_{n}} \int_{0}^{t_{n}} S(s) W_{n} w_{n} d s-S(s) \frac{1}{t_{n}} \int_{0}^{t_{n}} S(s) W_{n} w_{n} d s\right\| \\
& +\left\|S(s) \frac{1}{t_{n}} \int_{0}^{t_{n}} S(s) W_{n} w_{n} d s-S(s) w_{n}\right\| \\
\leq & 2\left\|w_{n}-\frac{1}{t_{n}} \int_{0}^{t_{n}} S(s) W_{n} w_{n} d s\right\| \\
& +\left\|\frac{1}{t_{n}} \int_{0}^{t_{n}} S(s) W_{n} w_{n} d s-S(s) \frac{1}{t_{n}} \int_{0}^{t_{n}} S(s) W_{n} w_{n} d s\right\| .
\end{aligned}
$$

It follows from (3.52) and (3.53) that

$$
\lim _{n \rightarrow \infty}\left\|w_{n}-S(s) w_{n}\right\|=0
$$

Indeed, from Lemma 2.9 and (3.55), we get $z \in F(\mathcal{S})$, that is, $z=S(s) z, \forall s \geq 0$.

(2) Next, we show that $z \in F(W)=\bigcap_{n=1}^{\infty} F\left(W_{n}\right)$, where $F\left(W_{n}\right)=\bigcap_{i=1}^{n} F\left(T_{i}\right), \forall n \geq 1$, and $F\left(W_{n+1}\right) \subset F\left(W_{n}\right)$. Assume that $z \notin F(W)$, then there exists a positive integer $m$ such that $z \notin F\left(T_{m}\right)$, and so $z \notin \bigcap_{i=1}^{m} F\left(T_{i}\right)$. Hence, for any $n \geq m, z \notin \bigcap_{i=1}^{n} F\left(T_{i}\right)=F\left(W_{n}\right)$, that is, $z \neq W_{n} z$. This together with $z=S(s) z, \forall s \geq 0$ shows that $z=S(s) z \neq S(s) W_{n} z, \forall s \geq 0$; therefore, we have $z \neq\left(1 / t_{n}\right) \int_{0}^{t_{n}} S(s) W_{n} z d s, \forall n \geq m$. It follows from the Opial's condition 
and (3.52) that

$$
\begin{aligned}
& \liminf _{i \rightarrow \infty}\left\|w_{n_{i}}-z\right\|<\liminf _{i \rightarrow \infty}\left\|w_{n_{i}}-\frac{1}{t_{n_{i}}} \int_{0}^{t_{n_{i}}} S(s) W_{n_{i}} z d s\right\| \\
& \leq \liminf _{i \rightarrow \infty}\left(\left\|w_{n_{i}}-\frac{1}{t_{n_{i}}} \int_{0}^{t_{n_{i}}} S(s) W_{n_{i}} w_{n_{i}} d s\right\|\right. \\
&\left.+\left\|\frac{1}{t_{n_{i}}} \int_{0}^{t_{n_{i}}} S(s) W_{n_{i}} w_{n_{i}} d s-\frac{1}{t_{n_{i}}} \int_{0}^{t_{n_{i}}} S(s) W_{n_{i}} z d s\right\|\right) \\
& \leq \liminf _{i \rightarrow \infty}\left\|w_{n_{i}}-z\right\|,
\end{aligned}
$$

which is a contradiction. Thus, we get $z \in F(W)$.

(3) Now, we prove that $z \in \operatorname{GMEP}\left(F_{1}, \varphi, A\right)$. Since $t_{n}=T_{r_{n}}^{\left(F_{1}, \varphi_{1}\right)}\left(x_{n}-r_{n} A x_{n}\right), n \geq 1$, we have for any $y \in C$ that

$$
F_{1}\left(t_{n}, y\right)+\varphi_{1}(y)-\varphi_{1}\left(t_{n}\right)+\left\langle A x_{n}, y-t_{n}\right\rangle+\frac{1}{r_{n}}\left\langle y-t_{n}, t_{n}-x_{n}\right\rangle \geq 0, \quad \forall y \in \mathrm{C}
$$

From (A2), we also have

$$
\varphi_{1}(y)-\varphi_{1}\left(t_{n}\right)+\left\langle A x_{n}, y-t_{n}\right\rangle+\frac{1}{r_{n}}\left\langle y-t_{n}, t_{n}-x_{n}\right\rangle \geq F_{1}\left(y, t_{n}\right), \quad \forall y \in \mathrm{C} .
$$

For $t$ with $0<t \leq 1$ and $y \in C$, let $y_{t}=t y+(1-t) z$. Since $y \in C$ and $z \in C$, we have $y_{t} \in C$. Then, we have

$$
\begin{aligned}
\left\langle y_{t}-t_{n_{i}}, A y_{t}\right\rangle \geq & \left\langle y_{t}-t_{n_{i}}, A y_{t}\right\rangle-\varphi_{1}\left(y_{t}\right)+\varphi_{1}\left(t_{n_{i}}\right)-\left\langle y_{t}-t_{n_{i}}, A x_{n_{i}}\right\rangle \\
& -\left\langle y_{t}-t_{n_{i}}, \frac{t_{n_{i}}-x_{n_{i}}}{r_{n_{i}}}\right\rangle+F_{1}\left(y_{t}, t_{n_{i}}\right) \\
= & \left\langle y_{t}-t_{n_{i}}, A y_{t}-A t_{n_{i}}\right\rangle+\left\langle y_{t}-t_{n_{i}}, A t_{n_{i}}-A x_{n_{i}}\right\rangle-\varphi_{1}\left(y_{t}\right)+\varphi_{1}\left(t_{n_{i}}\right) \\
& -\left\langle y_{t}-t_{n_{i}}, \frac{t_{n_{i}}-x_{n_{i}}}{r_{n_{i}}}\right\rangle+F_{1}\left(y_{t}, t_{n_{i}}\right) .
\end{aligned}
$$

Since $\left\|t_{n_{i}}-x_{n_{i}}\right\| \rightarrow 0$, we have $\left\|A t_{n_{i}}-A x_{n_{i}}\right\| \rightarrow 0$. Furthermore, from the inverse-strongly monotonicity of $A$, we have $\left\langle y_{t}-t_{n_{i}}, A y_{t}-A t_{n_{i}}\right\rangle \geq 0$. So, from (A4), (A5), and the weak lower 
semicontinuity of $\varphi_{1},\left(t_{n_{i}}-x_{n_{i}}\right) / r_{n_{i}} \rightarrow 0$ and $t_{n_{i}} \rightarrow z$, we have at the limit

$$
\left\langle y_{t}-z, A y_{t}\right\rangle \geq-\varphi_{1}\left(y_{t}\right)+\varphi_{1}(z)+F_{1}\left(y_{t}, z\right)
$$

as $i \rightarrow \infty$. From (A1), (A4), and (3.60), we also get

$$
\begin{aligned}
0 & =F_{1}\left(y_{t}, y_{t}\right)+\varphi_{1}\left(y_{t}\right)-\varphi_{1}\left(y_{t}\right) \\
& \leq t F_{1}\left(y_{t}, y\right)+(1-t) F_{1}\left(y_{t}, z\right)+t \varphi_{1}(y)-(1-t) \varphi_{1}(z)-\varphi\left(y_{t}\right) \\
& =t\left[F_{1}\left(y_{t}, y\right)+\varphi_{1}(y)-\varphi_{1}\left(y_{t}\right)\right]+(1-t)\left[F_{1}\left(y_{t}, z\right)+\varphi_{1}(z)-\varphi_{1}\left(y_{t}\right)\right] \\
& \leq t\left[F_{1}\left(y_{t}, y\right)+\varphi_{1}(y)-\varphi_{1}\left(y_{t}\right)\right]+(1-t)\left\langle y_{t}-z, A y_{t}\right\rangle \\
& =t\left[F_{1}\left(y_{t}, y\right)+\varphi_{1}(y)-\varphi_{1}\left(y_{t}\right)\right]+(1-t) t\left\langle y-z, A y_{t}\right\rangle,
\end{aligned}
$$

and hence,

$$
0 \leq F_{1}\left(y_{t}, y\right)+\varphi_{1}(y)-\varphi_{1}\left(y_{t}\right)+(1-t)\left\langle y-z, A y_{t}\right\rangle
$$

Letting $t \rightarrow 0$, we have, for each $y \in C$,

$$
F_{1}(z, y)+\varphi_{1}(y)-\varphi_{1}(z)+\langle y-z, A z\rangle \geq 0
$$

This implies that $z \in \operatorname{GMEP}\left(F_{1}, \varphi, A\right)$. By following the same arguments, we can show that $z \in \operatorname{GMEP}\left(F_{2}, \varphi, B\right)$.

(4) At last, we show that $z \in I\left(E_{2}, M_{2}\right)$. Infact, since $E_{2}$ is $\eta_{2}$-inverse-strongly monotone, this implies that $E_{2}$ is $\left(1 / \eta_{2}\right)$-Lipschitz continuous monotone mapping and domain of $E_{2}$ equal to $H$. It follows from Lemma 2.5 that $M_{2}+E_{2}$ is a maximal monotone. Let $(y, g) \in G\left(M_{2}+E_{2}\right)$, that is, $g-E_{2} y \in M_{2}(y)$. Since $w_{n_{i}}=J_{M_{2}, \lambda_{2}}\left(v_{n_{i}}-\lambda_{2} E_{2} v_{n_{i}}\right)$, we have $v_{n_{i}}-\lambda_{2} E_{2} v_{n_{i}} \in\left(I+\lambda_{2} M_{2}\right)\left(w_{n_{i}}\right)$, that is,

$$
\frac{1}{\lambda_{2}}\left(v_{n_{i}}-w_{n_{i}}-\lambda_{2} E_{2} v_{n_{i}}\right) \in M_{2}\left(w_{n_{i}}\right) .
$$

Since $M_{2}+E_{2}$ is a maximal monotone, we have

$$
\left\langle y-w_{n_{i}}, g-B y-\frac{1}{\lambda_{2}}\left(v_{n_{i}}-w_{n_{i}}-\lambda_{2} E_{2} v_{n_{i}}\right)\right\rangle \geq 0
$$


and so

$$
\begin{aligned}
\left\langle y-w_{n_{i}}, g\right\rangle & \geq\left\langle y-w_{n_{i}}, E_{2} y+\frac{1}{\lambda_{2}}\left(v_{n_{i}}-w_{n_{i}}-\lambda_{2} E_{2} v_{n_{i}}\right)\right\rangle \\
& =\left\langle y-w_{n_{i}}, E_{2} y-E_{2} w_{n_{i}}+E_{2} w_{n_{i}}-E_{2} v_{n_{i}}+\frac{1}{\lambda_{2}}\left(v_{n_{i}}-w_{n_{i}}\right)\right\rangle \\
& \geq 0+\left\langle y-w_{n_{i}}, E_{2} w_{n_{i}}-E_{2} v_{n_{i}}\right\rangle+\left\langle y-w_{n_{i}}, \frac{1}{\lambda_{2}}\left(v_{n_{i}}-w_{n_{i}}\right)\right\rangle .
\end{aligned}
$$

It follows from $\left\|v_{n}-w_{n}\right\| \rightarrow 0,\left\|E_{2} v_{n}-E_{2} w_{n}\right\| \rightarrow 0$, and $w_{n_{i}}-z$ that

$$
\lim _{i \rightarrow \infty}\left\langle y-w_{n_{i}}, g\right\rangle=\langle y-w, g\rangle \geq 0
$$

It follows from the maximal monotonicity of $M_{2}+E_{2}$ that $0 \in\left(M_{2}+E_{2}\right)(z)$, that is, $z \in$ $I\left(E_{2}, M_{2}\right)$. By following the same arguments, we can show that $z \in I\left(E_{1}, M_{1}\right)$. Hence, by (1)-(4), we have $z \in \Theta$.

Step 5. Noting that $x_{n}=P_{C_{n}} x_{0}$, by (2.5), we have

$$
\left\langle x_{0}-x_{n}, y-x_{n}\right\rangle \leq 0, \quad \forall y \in C_{n} .
$$

Since $\Theta \subset C_{n}$ and by the continuity of inner product, we obtain from the above inequality that

$$
\left\langle x_{0}-z, y-z\right\rangle \leq 0, \quad \forall y \in C
$$

By (2.5) again, we conclude that $\mathrm{z}=P_{\Theta} x_{0}$. This completes the proof.

Using Theorem 3.1, we obtain the following corollaries.

Corollary 3.2. Let $C$ be a nonempty closed-convex subset of a real Hilbert Space H. Let $F_{1}, F_{2}$ be bifunctions of $C \times C$ into real numbers $\mathbb{R}$ satisfying $(A 1)-(A 5)$, and let $\varphi_{1}, \varphi_{2}: C \rightarrow \mathbb{R} \cup\{+\infty\}$ be proper lower semicontinuous and convex functions with assumption (B1) or (B2). Let $A, B, E_{1}, E_{2}$ be $\alpha, \beta, \eta_{1}, \eta_{2}$-inverse-strongly monotone mappings of $C$ into $H$, respectively. Let $S=\{S(s): 0 \leq s<$ $\infty\}$ be a nonexpansive semigroup on $C$, and let $\left\{t_{n}\right\}$ be a positive real divergent sequence. Let $\left\{V_{i}\right.$ : $C \rightarrow C\}_{i=1}^{\infty}$ be a countable family of uniformly $k$-strict pseudocontractions, let $\left\{T_{i}: C \rightarrow C\right\}_{i=1}^{\infty}$ be a countable family of nonexpansive mappings defined by $T_{i} x=t x+(1-t) V_{i} x, \forall x \in C, \forall i \geq 1, t \in[k, 1)$, and let $W_{\mathrm{n}}$ be the $W$-mapping defined by (2.11) and $W$ a mapping defined by (2.12) with $F(W) \neq \emptyset$. 
Suppose that $\Theta:=F(\mathcal{S}) \cap F(W) \cap G M E P\left(F_{1}, \varphi_{1}, A\right) \cap G M E P\left(F_{2}, \varphi_{2}, B\right) \cap V I\left(C, E_{1}\right) \cap V I\left(C, E_{2}\right) \neq \emptyset$. Let $\left\{x_{n}\right\}$ be a sequence generated by $x_{0} \in C, C_{1, i}=C, C_{1}=\bigcap_{i=1}^{\infty} C_{1, i}, x_{1}=P_{C_{1}} x_{0}$, and

$$
\begin{gathered}
t_{n}=T_{r_{n}}^{\left(F_{1}, \varphi_{1}\right)}\left(x_{n}-r_{n} A x_{n}\right), \\
u_{n}=T_{q_{n}}^{\left(F_{2}, \varphi_{2}\right)}\left(t_{n}-q_{n} B t_{n}\right), \\
v_{n}=P_{C}\left(u_{n}-\lambda_{1} E_{1} u_{n}\right), \\
w_{n}=P_{C}\left(v_{n}-\lambda_{2} E_{2} v_{n}\right), \\
C_{n+1, i}=\left\{z \in C_{n, i}:\left\|y_{n, i}-z\right\|^{2} \leq\left\|x_{n}-z\right\|^{2}+\alpha_{n, i}\left(\left\|x_{0}\right\|^{2}+2\left\langle x_{n}-x_{0}, z\right\rangle\right)\right\}, \\
y_{n, i}=\alpha_{n, i} x_{0}+\left(1-\alpha_{n, i}\right) \frac{1}{t_{n}} \int_{0}^{t_{n}} S(s) W_{n} w_{n} d s, \\
C_{n+1}=\bigcap_{i=1}^{\infty} C_{n+1, i}, \\
x_{n+1}=P_{C_{n+1}} x_{0},
\end{gathered}
$$

for every $n \geq 0$, where $\left\{\alpha_{n, i}\right\}_{n=1}^{\infty} \subset(0,1),\left\{r_{n}\right\},\left\{q_{n}\right\} \subset(0, \infty), \lambda_{1} \in\left(0,2 \eta_{1}\right)$, and $\lambda_{2} \in\left(0,2 \eta_{2}\right)$ satisfy the following conditions:

(i) $0<a \leq r_{n} \leq b<2 \alpha$,

(ii) $0<c \leq q_{n} \leq d<2 \beta$,

(iii) $\lim _{n \rightarrow \infty} \alpha_{n, i}=0$,

(iv) $0<e \leq \lambda_{1} \leq f<2 \eta_{1}$,

(v) $0<g \leq \lambda_{2} \leq j<2 \eta_{2}$,

then $\left\{x_{n}\right\}$ converges strongly to $P_{\Theta} x_{0}$.

Proof. From Theorem 3.1, put $M=\partial \delta_{C}$, then $J_{M, \lambda_{1}}=P_{C}$ and $J_{M, \lambda_{2}}=P_{C}$. So we have $v_{n}=$ $P_{C}\left(u_{n}-\lambda_{1} E_{1} u_{n}\right)$ and $w_{n}=P_{C}\left(v_{n}-\lambda_{2} E_{2} v_{n}\right)$. The conclusion of Corollary 3.2 can be obtained from Theorem 3.1 immediately.

\section{Applications}

In this section, we study a kind of multiobjective optimization problem by using the result of this paper. We will give an iterative algorithm of solution for the following optimization problem with nonempty set of solutions:

$$
\begin{array}{cc}
\min & h_{1}(x) \\
\min & h_{2}(x) \\
& x \in \mathrm{C},
\end{array}
$$


where $h(x)$ is a convex and lower semicontinuous functional, and define C as a closedconvex subset of a real Hilbert space $H$. We denote the set of solutions of (4.1) by $M\left(h_{1}\right)$ and $M\left(h_{2}\right)$. Let $F_{i}: C \times C \rightarrow \mathbb{R}$ be a bifunction defined by $F_{i}(x, y)=h_{i}(y)-h_{i}(x)$. We consider the equilibrium problem, and it is obvious that $\operatorname{EP}\left(F_{i}\right)=M\left(h_{i}\right), i=1,2$. Therefore, from Theorem 3.1, we obtain the following Corollaries.

Corollary 4.1. Let $C$ be a nonempty closed-convex subset of a real Hilbert Space H. Let $h_{1}, h_{2}: C \rightarrow$ $\mathbb{R} \cup\{+\infty\}$ be proper lower semicontinuous and convex functions. Let $E_{1}, E_{2}$ be $\eta_{1}, \eta_{2}$-inverse-strongly monotone mappings of $C$ into $H$, respectively, and let $M_{1}, M_{2}: H \rightarrow 2^{H}$ be maximal monotone mappings. Let $\mathcal{S}=\{S(s): 0 \leq s<\infty\}$ be a nonexpansive semigroup on $C$, and let $\left\{t_{n}\right\}$ be a positive real divergent sequence. Let $\left\{V_{i}: C \rightarrow C\right\}_{i=1}^{\infty}$ be a countable family of uniformly $k$-strict pseudocontractions, let $\left\{T_{i}: C \rightarrow C\right\}_{i=1}^{\infty}$ be a countable family of nonexpansive mappings defined by $T_{i} x=t x+(1-t) V_{i} x, \forall x \in C, \forall i \geq 1, t \in[k, 1)$, and let $W_{n}$ be the $W$-mapping defined by (2.11) and $W$ a mapping defined by (2.12) with $F(W) \neq \emptyset$. Suppose that $\Theta:=F(\mathcal{S}) \cap F(W) \cap M\left(h_{1}\right) \cap M\left(h_{2}\right) \cap$ $I\left(E_{1}, M_{1}\right) \cap I\left(E_{2}, M_{2}\right) \neq \emptyset$. Let $\left\{x_{n}\right\}$ be a sequence generated by $x_{0} \in C, C_{1, i}=C, C_{1}=\bigcap_{i=1}^{\infty} C_{1, i}, x_{1}=$ $P_{C_{1}} x_{0}$, and

$$
\begin{gathered}
h_{1}(t)-h_{1}\left(t_{n}\right)+\frac{1}{r_{n}}\left\langle t-t_{n}, t_{n}-x_{n}\right\rangle \geq 0, \quad \forall t \in C, \\
h_{2}(u)-h_{2}\left(u_{n}\right)+\frac{1}{q_{n}}\left\langle u-u_{n}, u_{n}-t_{n}\right\rangle \geq 0, \quad \forall u \in C, \\
v_{n}=J_{M_{1}, \lambda_{1}}\left(u_{n}-\lambda_{1} E_{1} u_{n}\right), \\
w_{n}=J_{M_{2,}, \lambda_{2}}\left(v_{n}-\lambda_{2} E_{2} v_{n}\right), \\
C_{n+1, i}=\left\{z \in C_{n, i}:\left\|y_{n, i}-z\right\|^{2} \leq\left\|x_{n}-z\right\|^{2}+\alpha_{n, i}\left(\left\|x_{0}\right\|^{2}+2\left\langle x_{n}-x_{0}, z\right\rangle\right)\right\}, \\
y_{n, i}=\alpha_{n, i} x_{0}+\left(1-\alpha_{n, i}\right) \frac{1}{t_{n}} \int_{0}^{t_{n}} S(s) W_{n} w_{n} d s \\
C_{i=1}^{\infty} C_{n+1, i} \\
x_{n+1}=P_{C_{n+1}} x_{0}
\end{gathered}
$$

for every $n \geq 0$, where $\left\{\alpha_{n, i}\right\}_{n=1}^{\infty} \subset(0,1),\left\{r_{n}\right\},\left\{q_{n}\right\} \subset(0, \infty), \lambda_{1} \in\left(0,2 \eta_{1}\right)$, and $\lambda_{2} \in\left(0,2 \eta_{2}\right)$ satisfy the following conditions:

(i) $\liminf _{n \rightarrow \infty} r_{n}>0$,

(ii) $\liminf _{n \rightarrow \infty} q_{n}>0$,

(iii) $\lim _{n \rightarrow \infty} \alpha_{n, i}=0$,

(iv) $0<e \leq \lambda_{1} \leq f<2 \eta_{1}$,

(v) $0<g \leq \lambda_{2} \leq j<2 \eta_{2}$,

then $\left\{x_{n}\right\}$ converges strongly to $P_{\Theta} x_{0}$. 
Proof. From Theorem 3.1, put $F_{1}\left(t_{n}, t\right)=h_{1}(t)-h_{1}\left(t_{n}\right), F_{2}\left(u_{n}, u\right)=h_{2}(u)-h_{2}\left(u_{n}\right)$, and $A, B, \varphi_{1}, \varphi_{2} \equiv 0$. The conclusion of Corollary 4.1 can be obtained from Theorem 3.1 immediately.

Corollary 4.2. Let $C$ be a nonempty closed-convex subset of a real Hilbert Space H. Let $h_{1}, h_{2}: C \rightarrow$ $\mathbb{R} \cup\{+\infty\}$ be proper lower semicontinuous and convex functions. Let $E_{1}, E_{2}$ be $\eta_{1}, \eta_{2}$-inverse-strongly monotone mappings of $C$ into $H$, respectively, and let $M_{1}, M_{2}: H \rightarrow 2^{H}$ be maximal monotone mappings. Let $\mathcal{S}=\{S(s): 0 \leq s<\infty\}$ be a nonexpansive semigroup on $C$, and let $\left\{t_{n}\right\}$ be a positive real divergent sequence. Let $\left\{V_{i}: C \rightarrow C\right\}_{i=1}^{\infty}$ be a countable family of uniformly $k$-strict pseudocontractions, let $\left\{T_{i}: C \rightarrow C\right\}_{i=1}^{\infty}$ be a countable family of nonexpansive mappings defined by $T_{i} x=t x+(1-t) V_{i} x, \forall x \in C, \forall i \geq 1, t \in[k, 1)$, and let $W_{n}$ be the $W$-mapping defined by (2.11) and $W$ a mapping defined by (2.12) with $F(W) \neq \emptyset$. Suppose that $\Theta:=F(\mathcal{S}) \cap F(W) \cap M\left(h_{1}\right) \cap M\left(h_{2}\right) \cap$ $V I\left(C, E_{1}\right) \cap V I\left(C, E_{2}\right) \neq \emptyset$. Let $\left\{x_{n}\right\}$ be a sequence generated by $x_{0} \in C, C_{1, i}=C, C_{1}=\bigcap_{i=1}^{\infty} C_{1, i}, x_{1}=$ $P_{C_{1}} x_{0}$, and

$$
\begin{gathered}
h_{1}(t)-h_{1}\left(t_{n}\right)+\frac{1}{r_{n}}\left\langle t-t_{n}, t_{n}-x_{n}\right\rangle \geq 0, \quad \forall t \in C, \\
h_{2}(u)-h_{2}\left(u_{n}\right)+\frac{1}{q_{n}}\left\langle u-u_{n}, u_{n}-t_{n}\right\rangle \geq 0, \quad \forall u \in C, \\
v_{n}=P_{C}\left(u_{n}-\lambda_{1} E_{1} u_{n}\right), \\
w_{n}=P_{C}\left(v_{n}-\lambda_{2} E_{2} v_{n}\right), \\
C_{n+1, i}=\left\{z \in C_{n, i}:\left\|y_{n, i}-z\right\|^{2} \leq\left\|x_{n}-z\right\|^{2}+\alpha_{n, i}\left(\left\|x_{0}\right\|^{2}+2\left\langle x_{n}-x_{0}, z\right\rangle\right)\right\}, \\
y_{n, i}=\alpha_{n, i} x_{0}+\left(1-\alpha_{n, i}\right) \frac{1}{t_{n}} \int_{0}^{t_{n}} S(s) W_{n} w_{n} d s, \\
C_{i=1}^{\infty} C_{n+1, i},
\end{gathered}
$$

for every $n \geq 0$, where $\left\{\alpha_{n, i}\right\}_{n=1}^{\infty} \subset(0,1),\left\{r_{n}\right\},\left\{q_{n}\right\} \subset(0, \infty), \lambda_{1} \in\left(0,2 \eta_{1}\right)$, and $\lambda_{2} \in\left(0,2 \eta_{2}\right)$ satisfy the following conditions:

(i) $\liminf _{n \rightarrow \infty} r_{n}>0$,

(ii) $\liminf _{n \rightarrow \infty} q_{n}>0$,

(iii) $\lim _{n \rightarrow \infty} \alpha_{n, i}=0$,

(iv) $0<e \leq \lambda_{1} \leq f<2 \eta_{1}$,

(v) $0<g \leq \lambda_{2} \leq j<2 \eta_{2}$,

then $\left\{x_{n}\right\}$ converges strongly to $P_{\Theta} x_{0}$. 


\section{Acknowledgments}

The authors thank the referees for their appreciation, valuable comments, and suggestions. They would like to thank the Higher Education Research Promotion and National Research University Project of Thailand, Office of the Higher Education Commission for financial support. Furthermore, they would like to thank the Faculty of science (KMUTT) and the National Research Council of Thailand. This work was completed with the support of the National Research Council of Thailand (NRCT 2010-2011).

\section{References}

[1] F. E. Browder and W. V. Petryshyn, "Construction of fixed points of nonlinear mappings in Hilbert space," Journal of Mathematical Analysis and Applications, vol. 20, no. 2, pp. 197-228, 1967.

[2] K. Goebel and W. A. Kirk, Topics on Metric Fixed-Point Theory, Cambridge University Press, Cambridge, UK, 1990.

[3] H. Brézis, Opérateurs Maximaux Monotones et Semi-Groupes de Contractions dans les Espaces de Hilbert, North-Holland Mathematics Studies, no. 5. Notas de Mathemática no. 50, North-Holland, Amsterdam, The Netherlands, 1973.

[4] S.-S. Zhang, J. H. W. Lee, and C. K. Chan, "Algorithms of common solutions to quasi variational inclusion and fixed point problems," Applied Mathematics and Mechanics, vol. 29, no. 5, pp. 571-581, 2008.

[5] R. S. Burachik, J. O. Lopes, and G. J. P. da Silva, "An inexact interior point proximal method for the variational inequality problem," Computational and Applied Mathematics, vol. 28, no. 1, pp. 15-36, 2009.

[6] E. Blum and W. Oettli, "From optimization and variational inequalities to equilibrium problems," The Mathematics Student, vol. 63, pp. 123-145, 1994.

[7] O. Chadli, N. C. Wong, and J. C. Yao, "Equilibrium problems with applications to eigenvalue problems," Journal of Optimization Theory and Applications, vol. 117, no. 2, pp. 245-266, 2003.

[8] O. Chadli, S. Schaible, and J. C. Yao, "Regularized equilibrium problems with application to noncoercive hemivariational inequalities," Journal of Optimization Theory and Applications, vol. 121, no. 3, pp. 571-596, 2004.

[9] S. D. Flåm and A. S. Antipin, "Equilibrium programming using proximal-like algorithms," Mathematical Programming, Series B, vol. 78, no. 1, pp. 29-41, 1997.

[10] A. Moudafi and M. Thera, "Proximal and dynamical approaches to equilibrium problems," in Lecture note in Economics and Mathematical Systems, vol. 477, pp. 187-201, Springer, New York, NY, USA, 1999.

[11] Z. Wang and Y. Su, "Strong convergence theorems of common elements for equilibrium problems and fixed point problems in Banach paces," Journal of Applied Mathematics and Informatics, vol. 28, no. 3-4, pp. 783-796, 2010.

[12] R. Wangkeeree and R. Wangkeeree, "Strong convergence of the iterative scheme based on the extragradient method for mixed equilibrium problems and fixed point problems of an infinite family of nonexpansive mappings," Nonlinear Analysis: Hybrid Systems, vol. 3, no. 4, pp. 719-733, 2009.

[13] Y. Yao, M. Aslam Noor, S. Zainab, and Y.-C. Liou, "Mixed equilibrium problems and optimization problems," Journal of Mathematical Analysis and Applications, vol. 354, no. 1, pp. 319-329, 2009.

[14] Y. Yao, Y. J. Cho, and R. Chen, "An iterative algorithm for solving fixed point problems, variational inequality problems and mixed equilibrium problems," Nonlinear Analysis, Theory, Methods and Applications, vol. 71, no. 7-8, pp. 3363-3373, 2009.

[15] L. C. Zeng, S. Y. Wu, and J. C. Yao, "Generalized KKM theorem with applications to generalized minimax inequalities and generalized equilibrium problems," Taiwanese Journal of Mathematics, vol. 10, no. 6, pp. 1497-1514, 2006.

[16] P. Kumam, "A hybrid approximation method for equilibrium and fixed point problems for a monotone mapping and a nonexpansive mapping," Nonlinear Analysis: Hybrid Systems, vol. 2, no. 4, pp. 1245-1255, 2008.

[17] P. Kumam, "A new hybrid iterative method for solution of equilibrium problems and fixed point problems for an inverse strongly monotone operator and a nonexpansive mapping," Journal of Applied Mathematics and Computing, vol. 29, no. 1-2, pp. 263-280, 2009.

[18] J.-W. Peng and J.-C. Yao, "Strong convergence theorems of iterative scheme based on the extragradient method for mixed equilibrium problems and fixed point problems," Mathematical and Computer Modelling, vol. 49, no. 9-10, pp. 1816-1828, 2009. 
[19] Y. Shehu, "Iterative methods for family of strictly pseudocontractive mappings and system of generalized mixed equilibrium problems and variational inequality problems," Fixed Point Theory and Applications, vol. 2011, Article ID 852789, 21 pages, 2011.

[20] T. Shimizu and W. Takahashi, "Strong convergence to common fixed points of families of nonexpansive mappings," Journal of Mathematical Analysis and Applications, vol. 211, no. 1, pp. 71-83, 1997.

[21] C. Martinez-Yanes and H. K. Xu, "Strong convergence of the CQ method for fixed point iteration processes," Nonlinear Analysis, Theory, Methods and Applications, vol. 64, no. 11, pp. 2400-2411, 2006.

[22] H. Zhou, "Convergence theorems of fixed points for $\kappa$-strict pseudo-contractions in Hilbert spaces," Nonlinear Analysis, Theory, Methods and Applications, vol. 69, no. 2, pp. 456-462, 2008.

[23] K. Shimoji and W. Takahashi, "Strong convergence to common fixed points of infinite nonexpansive mappings and applications," Taiwanese Journal of Mathematics, vol. 5, no. 2, pp. 387-404, 2001.

[24] S. S. Chang, Variational Inequalities and Related Problems, Chongqing Publishing House, 2007.

[25] Z. Opial, "Weak convergence of the sequence of successive approximations for nonexpansive mappings," Bulletin of the American Mathematical Society, vol. 73, pp. 595-597, 1967.

[26] K. K. Tan and H. K. Xu, "The nonlinear ergodic theorem for asymptotically nonexpansive mappings in Banach spaces," Proceedings of the American Mathematical Society, vol. 114, no. 2, pp. 399-404, 1992. 


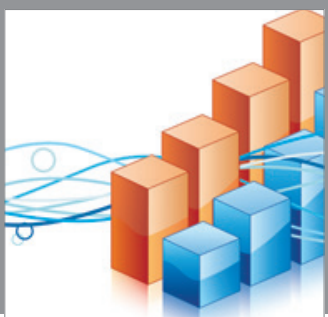

Advances in

Operations Research

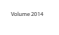

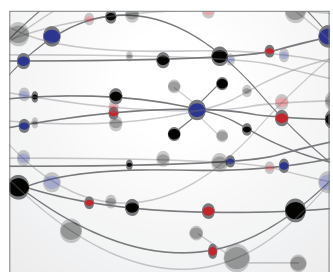

\section{The Scientific} World Journal
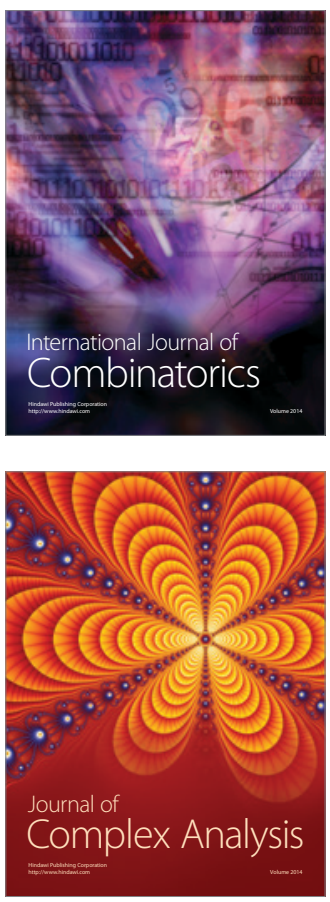

International Journal of

Mathematics and

Mathematical

Sciences
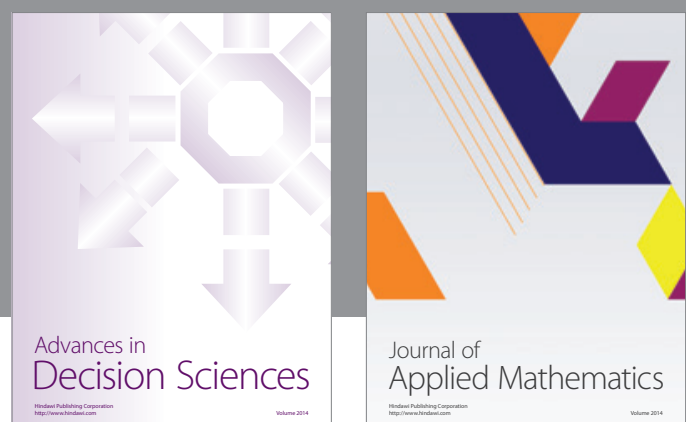

Journal of

Applied Mathematics
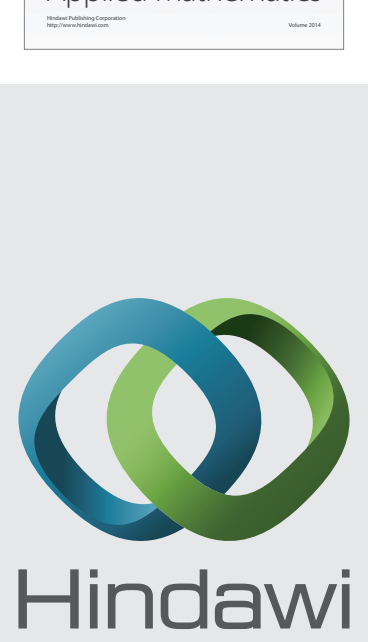

Submit your manuscripts at http://www.hindawi.com
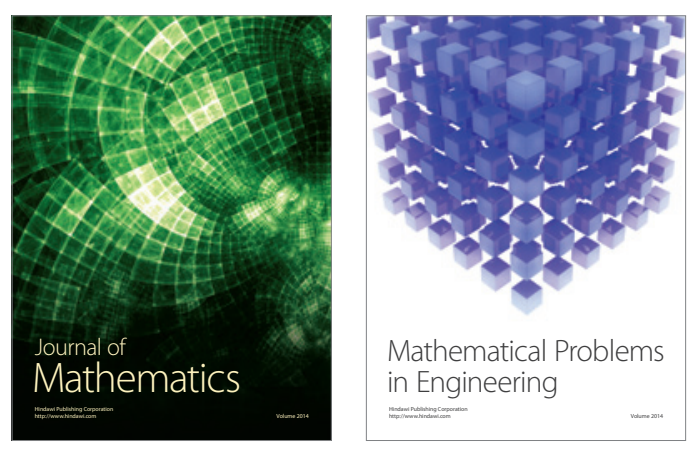

Mathematical Problems in Engineering
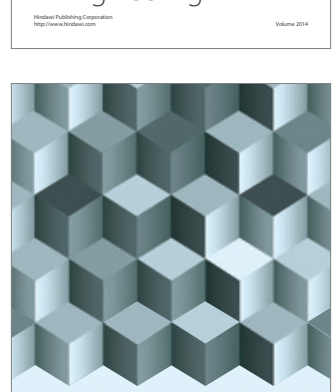

Journal of

Function Spaces
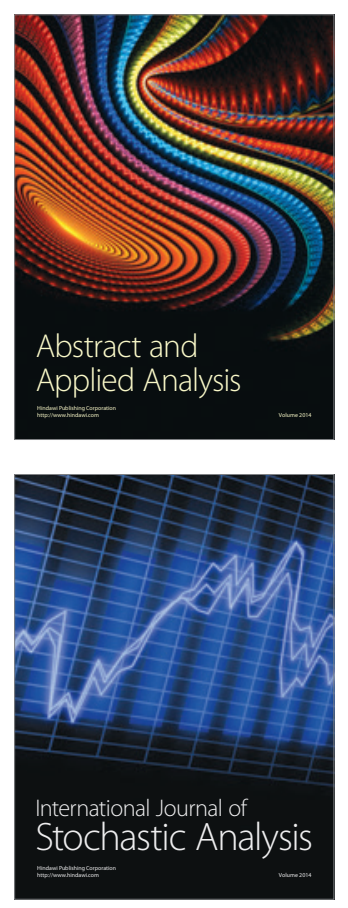

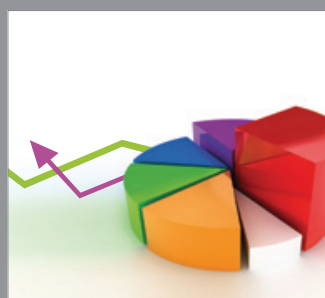

ournal of

Probability and Statistics

Promensencen
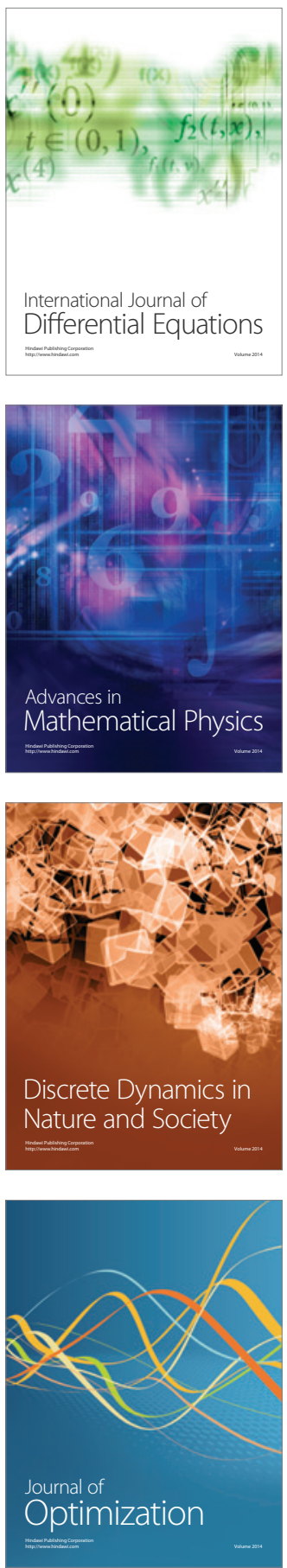